\title{
A Differential Algebraic Method for the Solution of the Poisson Equation for Charged Particle Beams
}

\author{
B. Erdelyi ${ }^{1, *}$, E. Nissen ${ }^{2}$ and S. Manikonda ${ }^{3}$ \\ ${ }^{1}$ Department of Physics, Northern Illinois University, DeKalb, IL, 60115, USA. \\ 2 European Organization for Nuclear Research, CH-1211 Geneva 23, Switzerland. \\ ${ }^{3}$ Advanced Magnet Lab, Palm Bay, FL, 32905, USA.
}

Received 24 August 2013; Accepted (in revised version) 17 June 2014

Communicated by Chi-Wang Shu

\begin{abstract}
The design optimization and analysis of charged particle beam systems employing intense beams requires a robust and accurate Poisson solver. This paper presents a new type of Poisson solver which allows the effects of space charge to be elegantly included into the system dynamics. This is done by casting the charge distribution function into a series of basis functions, which are then integrated with an appropriate Green's function to find a Taylor series of the potential at a given point within the desired distribution region. In order to avoid singularities, a Duffy transformation is applied, which allows singularity-free integration and maximized convergence region when performed with the help of Differential Algebraic methods. The method is shown to perform well on the examples studied. Practical implementation choices and some of their limitations are also explored.
\end{abstract}

PACS: 29.27.-a, 41.75.-i, 41.20.Cv, 05.10.-a

Key words: Charged particle beams, mean field limit, Poisson equation, orthogonal polynomials, differential algebra.

\section{Introduction}

Beams are ensembles of particles in directed motion. Charged particle beams underlie the science of particle accelerators with a variety of applications that span all science from physics to biology [1]. As accelerator technology improves, the usefulness of high intensity beams continues to increase, as illustrated by several existing and planned machines at the intensity frontier [2]. In order to properly design new accelerators and analyze

*Corresponding author. Email addresses: berdelyi@niu.edu (B. Erdelyi), nissen@jlab.org (E. Nissen), smanikonda@magnetlab.com (S. Manikonda) 
existing machines to improve performance, a fast and accurate method of modeling and simulating intense charged particle beams is required. Quantitatively, the phenomena arising from the average self-fields of beams, i.e. the electrostatic mean field limit, is called space charge. The beam dynamics that includes both externally applied fields as well as the space charge fields is paramount to understand in order to design better future machines and improve operational performance of existing machines. In order to determine these fields, we must solve Poisson's equation. With open boundary conditions it is,

$$
\nabla^{2} \Phi=-\frac{\rho}{\epsilon_{0}}
$$

where $\Phi$ is the scalar potential to vanish at infinity, and which is due to a smooth charge density distribution $\rho$.

There are several methods to perform these simulations, most popular being the particle in cell method [3]. Particle in cell methods involve solving the Poisson equation on a grid, usually aided by (fast) Fourier transform methods. It uses various charge deposition and force interpolation algorithms. Another alternative is to evolve in time the one-particle phase space distribution of the beams using the method of characteristics, or by the direct solution of the corresponding Vlasov equation in the six dimensional phase space. In any case, a requirement for accurate and efficient examination of the effects of space charge is a powerful Poisson solver. Examples of methods for calculating the potential are the Fourier transform methods [4] and wavelet methods [5]. Other methods involve fitting the charge distribution to a series of functions which are then used to determine the potential, one example of which is the Green's function method [6]. In fact, there is a long history in beam and accelerator physics related to Poisson solvers, including their comparison. A particularly illuminating summary is [7] and references therein. Here we detail our novel Poisson solver, for which preliminary results and applications can be found in [8-11].

Before we go further detailing our Poisson solver, it is important to mention another method that is extensively developed for single-particle beam dynamics: the transfer map method $[12,13]$. Transfer maps give the functional dependence between some final and initial conditions and contain all information about the system dynamics. In this context, normal form methods applied to transfer maps are powerful methods that allow a comprehensive analysis of low intensity beam dynamics [12,13]. In order to extend the method to include space charge dynamics self-consistently, clearly the beam self-potential needs to be specified. Since the transfer map method requires an analytic polynomial representation, the question arises how to obtain such a representation from a particle distribution. This paper shows that with the help of Differential Algebraic (DA) methods [13], and some innovative algorithmic developments presented here, it is possible to obtain high order Taylor expansions of the beam self-potential around a point that is situated inside the charge distribution. For the purpose of this paper, we will restrict ourselves to open boundary conditions. 
We emphasize that the novelty of the work presented in this paper stems from these two aspects: the ability, for the first time, to extract self-consistent transfer maps for space charge dominated beams, which requires a method to represent the scalar potential globally, over the spatial extent of the beam, in real analytic form from which a truncated Taylor expansion can be extracted. No previous Poisson solver employed in accelerator physics has this property. We rectify this here. Our results are compared with the exact, direct point-to-point, method instead of some other approximate method. The details of the map extraction method, examples, and its applications will be presented in a forthcoming publication. Some preliminary results can be found in [8].

Therefore, this work can be regarded as a novel Poisson solver that may find widespread applications wherever the need for such a solver arises. The new methods have been implemented into the general purpose nonlinear dynamics software package COSY Infinity [14], which can create non-linear Taylor transfer maps using DA techniques. A discussion of some DA basics and applications necessary for this paper is found in Appendix A.

The main elements of the overall algorithm are detailed in the following sections, as follows: Section 2 describes the method used to numerically calculate the potential from an analytic distribution function, Section 3 looks at the various ways of determining the distribution function based on a series of test particles, Section 4 describes the ways the method is put into practice, Section 5 examines the numerical concerns involved, as well as a benchmarking example, and finally Section 6 is a summary of the work.

\section{Numerically calculating the potential from an arbitrary analytic distribution}

In an ideal world, assuming the distribution of charge is analytic and known, the potential is easily found by multiplying the distribution with a dimensionally appropriate Green's function and integrating. However, if the point where the value of the potential is desired is within the distribution itself, the fact that there is a singularity at the evaluation point makes numerical integration of the distribution problematic. If we were interested in finding just the value of the potential at a point this could be solved through judiciously choosing the bounds of integration and the evaluation points. However, since we would like to compute the potential throughout the region occupied by a charged particle beam, such methods would shrink the region of convergence of the resulting Taylor series unacceptably. See Appendix A for details about DA integration.

The solution to this problem is to use what is known as a Duffy transformation [15]. This is a series of coordinate transforms which split the integral up into a sum of integrals over smaller domains such that the singularity is removed in the end. This allows the integral to be computed with quadratures having any nodes, allowing for an accurate determination of the potential. Again, if the integral is computed by employing the DA techniques detailed in Appendix A, the result is not only the value of the integral, but also 
its Taylor expansion with respect to any user-specified parameters. If these parameters are set to be the evaluation point for the potential, the result is the Taylor expansion of the potential around that point. The process is described in some details below in two dimensions, followed by some examples in later sections. The three dimensional case can be found in Appendix C.

\subsection{Two dimensional Duffy transformation}

Typically, charged particle beams have compactly supported unimodal charge distributions. If an analytic charge distribution function is denoted by $\rho$ and the potential is evaluated at the point $\left(x_{0}, y_{0}\right)$ inside its support, the value of the integral

$$
I\left(x_{0}, y_{0}\right)=\int_{c}^{d} \int_{a}^{b} \rho(x, y) \ln \left(\sqrt{\left(x-x_{0}\right)^{2}+\left(y-y_{0}\right)^{2}}\right) d x d y,
$$

is the potential at that point. We assume that the support of $\rho$ is inside the rectangle $[a, b] \times[c, d]$ and we are interested in the potential inside this region.

First, the rectangle is subdivided into four rectangles as shown in Fig. 1, which moves the singularity from the interior of the domain to the corners of four regions.

Due to this domain split, the total integral is transformed into four integrals which can have their limits of integration rearranged such that the equation becomes

$$
\begin{aligned}
I= & \int_{y_{0}}^{c} \int_{x_{0}}^{a} \rho(x, y) \ln \left(\sqrt{\left(x-x_{0}\right)^{2}+\left(y-y_{0}\right)^{2}}\right) d x d y \\
& -\int_{y_{0}}^{c} \int_{x_{0}}^{b} \rho(x, y) \ln \left(\sqrt{\left(x-x_{0}\right)^{2}+\left(y-y_{0}\right)^{2}}\right) d x d y \\
& -\int_{y_{0}}^{d} \int_{x_{0}}^{a} \rho(x, y) \ln \left(\sqrt{\left(x-x_{0}\right)^{2}+\left(y-y_{0}\right)^{2}}\right) d x d y \\
& +\int_{y_{0}}^{d} \int_{x_{0}}^{b} \rho(x, y) \ln \left(\sqrt{\left(x-x_{0}\right)^{2}+\left(y-y_{0}\right)^{2}}\right) d x d y .
\end{aligned}
$$

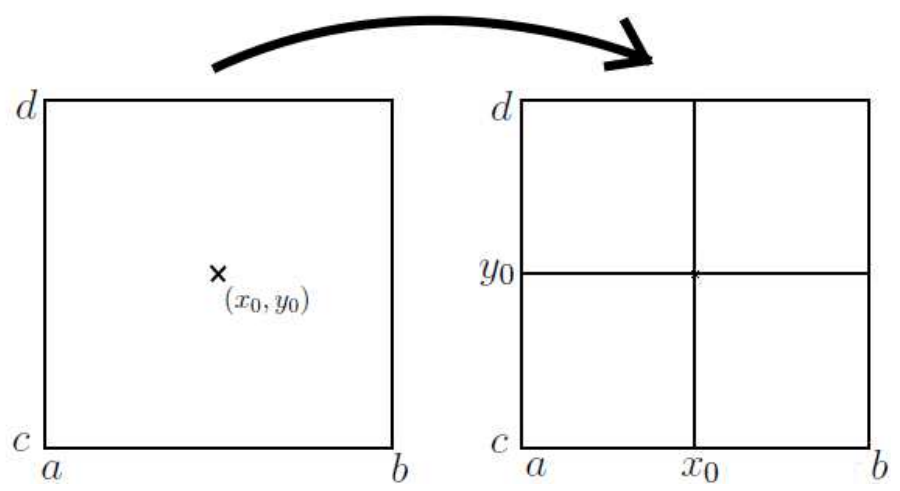

Figure 1: The full integration region is subdivided into four smaller regions with one of their corners on the expansion point. 


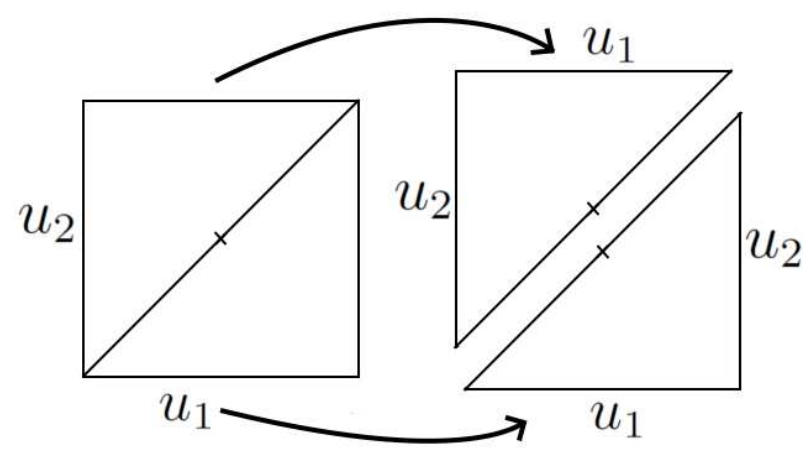

Figure 2: This shows how the integration region is further subdivided into triangles.

These are now all the same kind of integrals, merely with different bounds of integration. Henceforth we will look at the first integral in the series, remembering that all four will be solved in the same way. We continue by rescaling the rectangles to unit squares by the coordinate transformation:

$$
\begin{aligned}
u_{1} & =\frac{x-x_{0}}{a-x_{0}}, \\
u_{2} & =\frac{y-y_{0}}{c-y_{0}}, \\
d x & =\left(a-x_{0}\right) d u_{1}, \\
d y & =\left(c-y_{0}\right) d u_{2} .
\end{aligned}
$$

For expedience we will use $\lambda_{1}=\left(a-x_{0}\right)$ and $\lambda_{2}=\left(c-y_{0}\right)$. The first integral in (2.2) now becomes,

$$
I_{a c}=\int_{0}^{1} \int_{0}^{1} \lambda_{1} \lambda_{2} \rho\left(\lambda_{1} u_{1}+x_{0}, \lambda_{2} u_{2}+y_{0}\right) \ln \left(\sqrt{\lambda_{1}^{2} u_{1}^{2}+\lambda_{2}^{2} u_{2}^{2}}\right) d u_{1} d u_{2}
$$

As shown in Fig. 2, in order to perform the final coordinate transform, we cut the square into two triangles that are integrated separately,

$$
\begin{aligned}
I_{a c}= & \int_{0}^{1} \int_{0}^{u_{1}} \lambda_{1} \lambda_{2} \rho\left(\lambda_{1} u_{1}+x_{0}, \lambda_{2} u_{2}+y_{0}\right) \ln \left(\sqrt{\lambda_{1}^{2} u_{1}^{2}+\lambda_{2}^{2} u_{2}^{2}}\right) d u_{1} d u_{2} \\
& +\int_{0}^{u_{2}} \int_{0}^{1} \lambda_{1} \lambda_{2} \rho\left(\lambda_{1} u_{1}+x_{0}, \lambda_{2} u_{2}+y_{0}\right) \ln \left(\sqrt{\lambda_{1}^{2} u_{1}^{2}+\lambda_{2}^{2} u_{2}^{2}}\right) d u_{1} d u_{2} .
\end{aligned}
$$

In order to finish the Duffy transformation the triangles are now converted to squares yet again. This is accomplished with the transformation

$$
\begin{aligned}
& u_{1}=w_{1} \\
& u_{2}=w_{1} w_{2}
\end{aligned}
$$


in the first integral in (2.8), which makes the integral take the following form:

$$
\int_{0}^{1} \int_{0}^{1} \lambda_{1} \lambda_{2} w_{1} \rho\left(\lambda_{1} w_{1}+x_{0}, \lambda_{2} w_{1} w_{2}+y_{0}\right) \ln \left(\sqrt{\lambda_{1}^{2} w_{1}^{2}+\lambda_{2}^{2} w_{1}^{2} w_{2}^{2}}\right) d w_{1} d w_{2}
$$

This is simplified to

$$
\lambda_{1} \lambda_{2} \int_{0}^{1} \int_{0}^{1} \rho\left(\lambda_{1} w_{1}+x_{0}, \lambda_{2} w_{1} w_{2}+y_{0}\right)\left(w_{1} \ln \left(w_{1}\right)+w_{1} \ln \left(\sqrt{\lambda_{1}^{2}+\lambda_{2}^{2} w_{2}^{2}}\right)\right) d w_{1} d w_{2}
$$

Since $\lim _{w_{1} \rightarrow 0} w_{1} \ln \left(w_{1}\right)=0$, and the argument of the other logarithm is never zero, the singularity is removed. A similar transform is made to the second triangle (and the second term in (2.8)),

$$
\begin{aligned}
& u_{1}=w_{1} w_{2} \\
& u_{2}=w_{2}
\end{aligned}
$$

converting it into the following:

$$
\lambda_{1} \lambda_{2} \int_{0}^{1} \int_{0}^{1} \rho\left(\lambda_{1} w_{1} w_{2}+x_{0}, \lambda_{2} w_{2}+y_{0}\right)\left(w_{2} \ln \left(w_{2}\right)+w_{2} \ln \left(\sqrt{\lambda_{2}^{2}+\lambda_{1}^{2} w_{1}^{2}}\right)\right) d w_{1} d w_{2} .
$$

Assembling the partial results, we finally obtain a singularity-free formula for the computation of the potential,

$$
\begin{aligned}
\phi\left(x_{0}, y_{0}\right) & =\sum_{n=1}^{4} \lambda_{1, n} \lambda_{2, n} \int_{0}^{1} \int_{0}^{1} d w_{1} d w_{2} \\
\times & {\left[\begin{array}{c}
\rho\left(\lambda_{1, n} w_{1}+x_{0}, \lambda_{2, n} w_{1} w_{2}+y_{0}\right)\left(w_{1} \ln \left(w_{1}\right)+w_{1} \ln \left(\sqrt{\lambda_{1, n}^{2}+\lambda_{2, n}^{2} w_{2}^{2}}\right)\right) \\
+\rho\left(\lambda_{1, n} w_{1} w_{2}+x_{0}, \lambda_{2, n} w_{2}+y_{0}\right)\left(w_{2} \ln \left(w_{2}\right)+w_{2} \ln \left(\sqrt{\lambda_{2, n}^{2}+\lambda_{1, n}^{2} w_{1}^{2}}\right)\right)
\end{array}\right] . }
\end{aligned}
$$

It is shown in Appendix B that, if the original domain $[a, b] \times[c, d]$ is a square, the integrals only need to be determined once for a polynomial approximation of $\rho$ and can be scaled to fit the required bounds of integration and specific distribution very easily. The Duffy transformation works also in three dimensions, as shown in Appendix C.

As already mentioned, if the integral (2.15) is evaluated in DA, the result is not just the potential at $\left(x_{0}, y_{0}\right)$ but also the Taylor expansion of the potential around the point $\left(x_{0}, y_{0}\right)$. The question then arises about the region of convergence of the expansion. If we look at the final set of integrals (2.15) that need to be determined, and assume that the function $\rho$ does not limit the convergence region, it is clear that the domain of convergence is limited by the smallest value of $\lambda_{1, n}$ or $\lambda_{2, n}$ for which any of the square roots vanish. Since the domain of both $w_{1}$ and $w_{2}$ is $[0,1]$ it is easy to see that the radius of convergence $R$ is

$$
R=\min \left(b-x_{0}, x_{0}-a, d-y_{0}, y_{0}-c\right),
$$


equal to the distance between the expansion point and the nearest boundary of the rectangle $[a, b] \times[c, d]$. The point $\left(x_{0}, y_{0}\right)$ is typically a reference particle, used to set the origin of a local coordinate system, so without loss of generality can be set to $(0,0)$. Therefore, for best convergence we choose the smallest $a=b=c=d$ possible (determined by the charge distribution support), which is of course application dependent. More about this in Section 5.5. With this choice, the integration domain is a square and the scaling relations of Appendix B can be readily applied.

It is worthwhile mentioning that the resulting Taylor series in two or three variables can be reordered and regrouped into a series of homogeneous polynomials. It is wellknown that the resulting region of convergence can never be smaller than that given by (2.16), and indeed often it is a larger star-shaped domain. This is what we observe in practice too, as shown in the following sections. In this paper all integrals were determined using an eighth order Runge-Kutta method with seventh order automatic step size control, which is built into the code COSY Infinity [14].

\section{Calculating the distribution function from test particles}

In practice, often an analytically defined distribution function might not be known. In these cases, the important question of density estimation comes up. Here we explore density estimation based on expansion in some basis functions. Some basis functions will be more useful than others depending on the actual distribution; here we examine a sum of monomials and a sum of Legendre polynomials as the basis functions. Clearly, these are not the only expansions that can be used. They were chosen for demonstration purposes, and for a systematic study of density estimation for charged particle beams in orthogonal function bases see [11] and references therein.

If the distribution function is a sum of monomials, the potential will be a sum of the monomials integrated with a Green's function. As shown in Appendix B, the integrals can be precomputed and stored, and then read and scaled without reintegration at runtime, allowing for faster calculations.

\subsection{Smooth distribution functions}

We assume that there is a smooth function $f$ that describes the distribution, and that this function can be expanded in a complete set of smooth basis functions $\varphi$ of the form,

$$
f(x, y)=\sum_{i=0}^{\infty} \sum_{j=0}^{\infty} D_{i j} \varphi_{i}(x) \varphi_{j}(y)
$$

with coefficients $D_{i j}$. The task is to choose some specific basis functions and to determine the corresponding coefficients based on some limited information available about $f$. Typically, the available information are the moments of $f$. The moments may be known 
exactly to all orders, or only approximately to some finite order. The challenge is to extract the coefficients $D_{i j}$ as accurately as possible. There are several ways that this can be done. Here we examine two methods. One method involves calculating the moments of the distribution to find a sum of monomials, the other involves casting the distribution as a series of projections onto orthogonal polynomials.

\subsubsection{The moment method}

In this method we assume that the distribution function can be approximated using a series of the form

$$
\rho(x, y)=\sum_{i+j \leq n} C_{i j} x^{i} y^{j}
$$

According to [16], if two compactly supported distributions have the same moments, then they are mathematically identical. Hence, the goal of this density estimation method is to generate a polynomial approximation of the true density that has the same moments up to a fixed order as $f$. If the moments up to a given order are known, the series coefficients up to the same order can be determined. The question arises about the consequences due to the finite truncation order. Intuitively, the higher the truncation order, the closer the resemblance between the two functions is going to be. Nevertheless, the answers will still not be unique. However, it can be shown that among all single variable probability density functions $\rho$ with the same first $2 p$ moments as $f$ satisfy the relation [17]

$$
|f(x)-\rho(x)| \leq \frac{1}{\mathbf{V}_{p}^{T}(x) \mathbf{M}_{p}^{-1} \mathbf{V}_{p}(x)},
$$

where $\mathbf{V}_{p}^{T}(x)=\left(1, x, x^{2}, \cdots, x^{p}\right)$ and

$$
\mathbf{M}_{p}=\left(\begin{array}{cccc}
m_{1} & m_{2} & \cdots & m_{p} \\
m_{2} & m_{3} & \cdots & m_{p+1} \\
m_{3} & m_{4} & \cdots & m_{p+2} \\
\vdots & \vdots & \vdots & \vdots \\
m_{p+1} & m_{p+2} & \cdots & m_{2 p}
\end{array}\right),
$$

$m_{i}$ being the moments that the functions have in common. Thus, the tail probabilities will become indistinguishable first, followed by core probabilities. Convergence speed scales with $x^{-2 p}$. This is useful in providing practical guidance about the truncation orders to be employed in practice.

The moments are defined as

$$
M_{n m}=\int_{x_{\ell}}^{x_{u}} \int_{y_{\ell}}^{y_{u}} x^{n} y^{m} f(x, y) d x d y,
$$

as integrals over the support of $f$, where $x_{\ell}, x_{u}, y_{\ell}$, and $y_{u}$ are the horizontal lower and upper bounds, and the vertical lower and upper bounds of integration, respectively. 
The coefficients $C_{i j}$ are found by solving a linear system of equations that result from the matching conditions between the exact moments $M_{n m}$ and the moments of $\rho$. This is done by creating a system of equations such that,

$$
M_{n m}=\int_{-x_{r}}^{x_{r}} \int_{-y_{r}}^{y_{r}} x^{n} y^{m} \sum_{i} \sum_{j} C_{i j} x^{i} y^{j} d x d y,
$$

where we assume $\left|x_{\ell}\right|=x_{u}=x_{r}$ and $\left|y_{\ell}\right|=y_{u}=y_{r}$, which can be reduced to

$$
M_{n m}=\sum_{i} \sum_{j} N_{(n+i)(m+j)}\left(x_{r}, y_{r}\right) C_{i j}
$$

where

$$
N_{k l}(x, y)=\frac{x^{k+1} y^{l+1}}{(k+1)(l+1)}\left(\left(1-(-1)^{k+1}\right)\left(1-(-1)^{l+1}\right)\right) .
$$

This can be cast as a matrix equation $\vec{M}=N \vec{C}$, where $\vec{M}$ is a vector built from the moments $M_{n m}$ and $\vec{C}$ is a vector built from the coefficients $C_{i j}$. An example of how this is done is shown in Algorithm 3. The matrix $N$ is arranged in the following manner, where $p$ is the maximum order,

$$
\left(\begin{array}{c}
M_{00} \\
M_{10} \\
\vdots \\
M_{01} \\
\vdots \\
M_{0 p}
\end{array}\right)=\left(\begin{array}{cccccc}
N_{00}\left(x_{r}, y_{r}\right) & N_{10}\left(x_{r}, y_{r}\right) & \ldots & N_{01}\left(x_{r}, y_{r}\right) & \ldots & N_{0 p}\left(x_{r}, y_{r}\right) \\
N_{10}\left(x_{r}, y_{r}\right) & \cdots & N_{11}\left(x_{r}, y_{r}\right) & \cdots & N_{1 p-1}\left(x_{r}, y_{r}\right) & 0 \\
\vdots & & & & & \vdots \\
N_{01}\left(x_{r}, y_{r}\right) & N_{11}\left(x_{r}, y_{r}\right) & \ldots & N_{0 p}\left(x_{r}, y_{r}\right) & \ldots & 0 \\
\vdots & & & & & \vdots \\
N_{0 p}\left(x_{r}, y_{r}\right) & 0 & & & 0 & 0
\end{array}\right)\left(\begin{array}{c}
C_{00} \\
C_{10} \\
\vdots \\
C_{01} \\
\vdots \\
C_{0 p}
\end{array}\right) .
$$

The coefficients are solved by inverting $N$ and multiplying by the known moments, $C=N^{-1} M$. Assuming the region of integration is the square $[-1,1]$ (as justified in the preceding section) the matrix $N$ has the form,

$$
N=\left(\begin{array}{cccccccc}
1 & 0 & \frac{1}{3} & 0 & \frac{1}{5} & 0 & \cdots & \frac{1}{p+1} \\
0 & \frac{1}{3} & 0 & \frac{1}{5} & 0 & \cdots & \frac{1}{p+1} & 0 \\
\frac{1}{3} & 0 & \frac{1}{5} & 0 & \cdots & \frac{1}{p+1} & 0 & \vdots \\
0 & \frac{1}{5} & 0 & \cdots & \frac{1}{p+1} & 0 & & \vdots \\
\frac{1}{5} & 0 & \cdots & \frac{1}{p+1} & 0 & & & \vdots \\
0 & \cdots & \frac{1}{p+1} & 0 & & \ddots & & \vdots \\
\vdots & \frac{1}{p+1} & 0 & & & & \ddots & \vdots \\
\frac{1}{p+1} & 0 & \cdots & \cdots & \cdots & \cdots & \cdots & 0
\end{array}\right) .
$$


This is an ill-conditioned matrix for high values of $p$. In order to preserve as much accuracy as possible a truncated singular value decomposition method [18] was adapted for our use. In summary, this method is a useful way of estimating distributions as Taylor series since it only requires that the statistical moments of the distributions through some fixed finite order be known.

\subsubsection{Orthogonal polynomials}

Since the distribution has compact support, at least any of the Jacobi polynomials could be used for the purpose of distribution estimation. For the scope of this paper and for simplicity we present the use of Legendre polynomials $P_{i}(x)$.

If we assume that the distribution can be expanded as,

$$
\rho(x, y)=\sum_{i+j \leq n} \tilde{C}_{i j} P_{i}(x) P_{j}(y)
$$

and knowing that Legendre polynomials satisfy the orthogonality condition,

$$
\int_{-1}^{1} P_{\ell}(x) P_{m}(x) d x=\frac{2}{2 \ell+1} \delta_{\ell m} .
$$

The coefficients can be calculated from

$$
\int_{-1}^{1} \int_{-1}^{1} P_{i}(x) P_{j}(y) \rho(x, y) d x d y=\tilde{C}_{i j} \frac{2}{2 i+1} \frac{2}{2 j+1} .
$$

We are essentially calculating the Legendre polynomial moments of the distribution instead of the monomial moments used in the moment method. On the other hand, if we assume we already know the monomial moments, it is easy to calculate the Legendre coefficients directly, as follows. The same moment matching conditions are applied, that is, we require that the moments of $\rho$ are the same as the actual moments of $f$ up to a fixed order $n$. Since every product of Legendre polynomials can be re-expanded into a power series,

$$
P_{i}(x) P_{j}(y)=\sum_{k+l \leq i+j} a_{k l} x^{k} y^{l}
$$

the left hand side of (3.13) can be written as a linear combination of these moments, and we obtain

$$
\tilde{C}_{i j}=\frac{(2 i+1)(2 j+1)}{4} \vec{A} \cdot \vec{M}
$$

where $\vec{A}$ is a coefficient vector built from $a_{k l}$. Hence, we obtain an approximation of the original distribution as a series of Legendre polynomials without the need to invert any matrices. 


\subsection{Estimating smooth distributions from discrete point distributions}

The previous examples assumed that the moments of a distribution were known exactly. However, that is not always the case in practice. In fact, when studying particle systems, a finite number of test particles are used to model the beam. The main difference in this case is that $\rho(x, y)$ is no longer a smooth function but is now a collection of particles, i.e. can be described by a discrete point distribution in terms of a sum of Dirac delta functions,

$$
\rho(x, y)=\sum_{i=1}^{N} \delta\left(x-x_{i}\right) \delta\left(y-y_{i}\right),
$$

where the sum is over the number of particles $N$ and $\left(x_{i}, y_{i}\right)$ are the individual particle coordinates in configuration space. The moments of this distribution are calculated by

$$
M_{n m}=\sum_{i=1}^{N} x_{i}^{n} y_{i}^{m} .
$$

When (3.17) is utilized in (3.9), we obtain the discrete variant of the moment method. The calculation of Legendre coefficients takes the form,

$$
\tilde{C}_{i j}=\frac{2 i+1}{2} \frac{2 j+1}{2} \sum_{n=1}^{N} P_{i}\left(x_{n}\right) P_{j}\left(y_{n}\right),
$$

by directly applying (3.16) to (3.13).

These changes do not alter the methods used to find the potential, but they might alter what methods will finally be used for simulation purposes, as discussed in Section 5.6 .

\section{Implementation}

The main ingredient of the equations of the motion of self-interacting charged particle system dynamics is the electric field caused by the space charge. This is obtained from the potential, by the gradient operator. Taking the gradient in DA is an elementary operation, in which no truncation or interpolation error is involved. Therefore, the potential computation requires most effort. This section describes the main aspects of the implementation of the computation of the Taylor expansion of the potential using the methods described in the preceding sections and for a variety of cases and circumstances.

\subsection{Using the moment method}

Schematically, the new method of obtaining the scalar potential is outlined in Fig. 3.

Using the moment method involves the determination of the optimum order for moment calculation and of the range of integration (i.e. the support of the distribution function). A simple outline of the method is shown in Algorithms 1-4. 


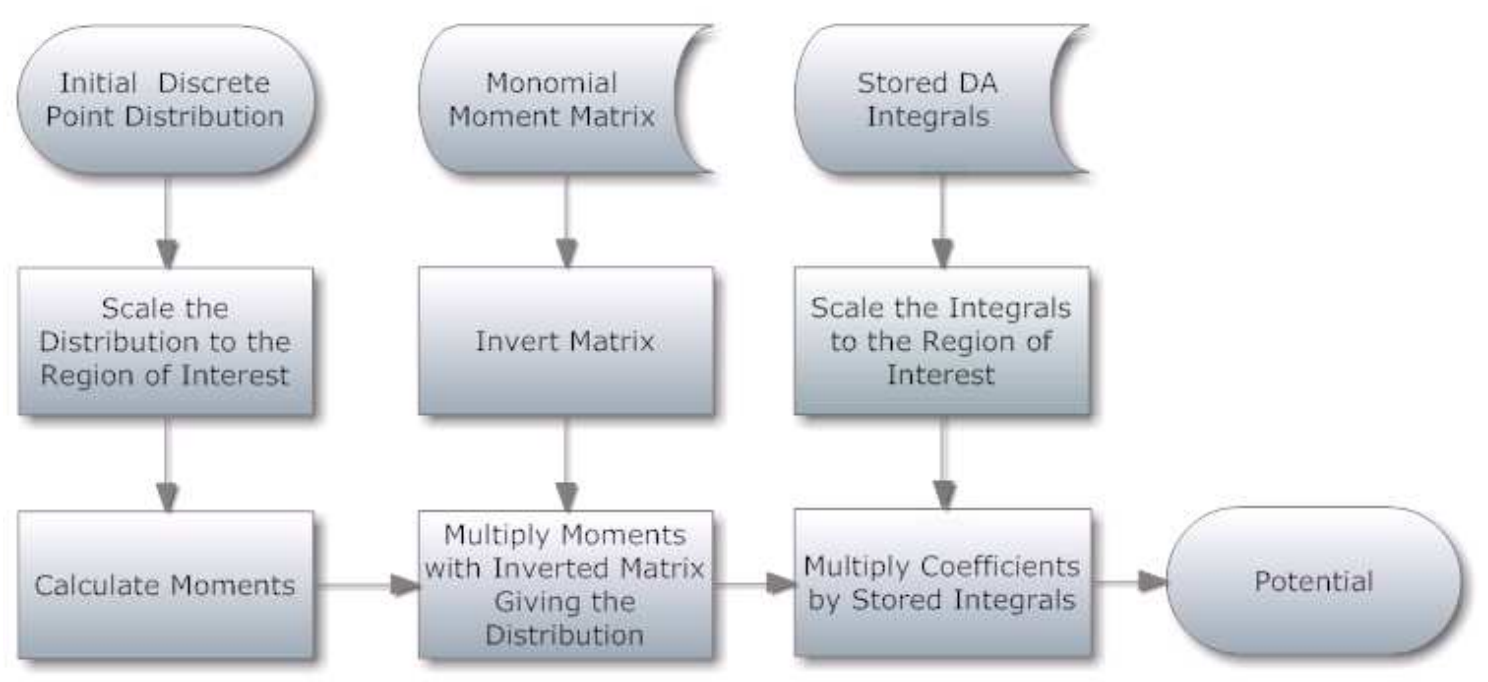

Figure 3: Overview of the method used to find the potential of a given distribution.

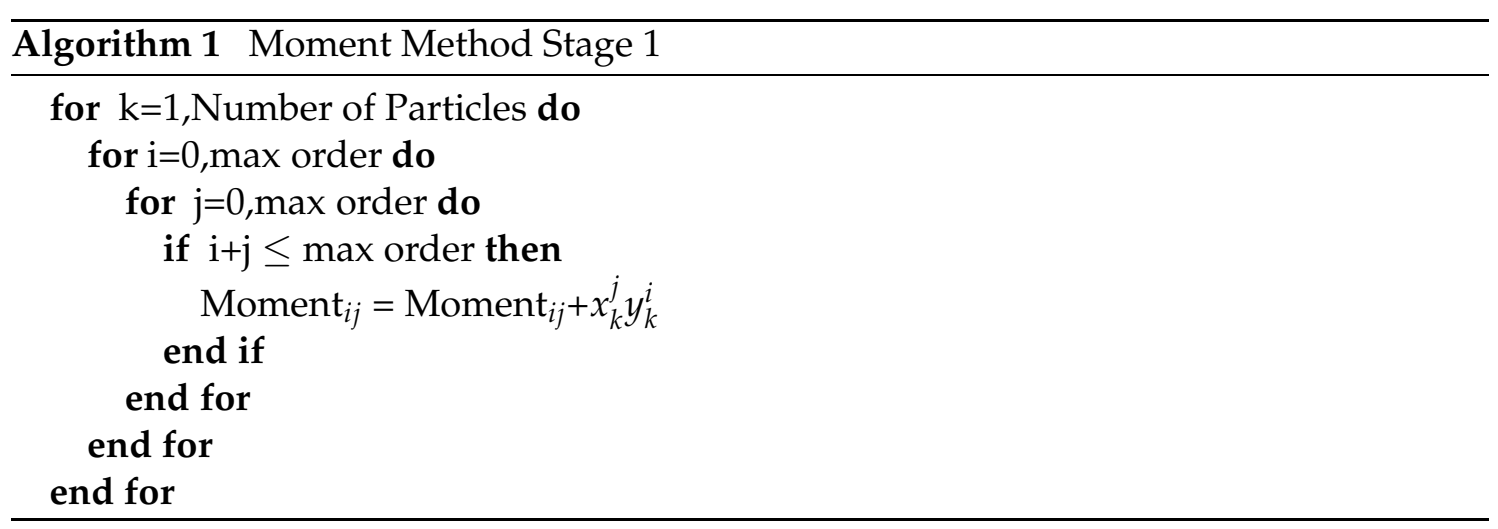

As illustrated in Algorithm 1, stage 1 of the moment method creates the moment matrix obtained from (3.5). Since we are assuming that the distribution is a collection of delta functions, the moment integrals are just a sum of the relevant powers of the coordinates.

Stage 2 of the moment method creates the transfer matrix between moments and the Taylor coefficients (Algorithm 2). The matrix is then inverted using truncated SVD inversion. The order of the indices is arranged such that the matrix entries will match with the result of Stage 3 in Algorithm 3.

Stage 3 of the moment method transfers a matrix of calculated moments into a vector which can be multiplied by the inverted matrix found in Stage 2 to find the coefficient vector for the Taylor series representing the distribution function of the particles.

Stage 4 determines the potential by combining the Taylor series coefficients with pre- 

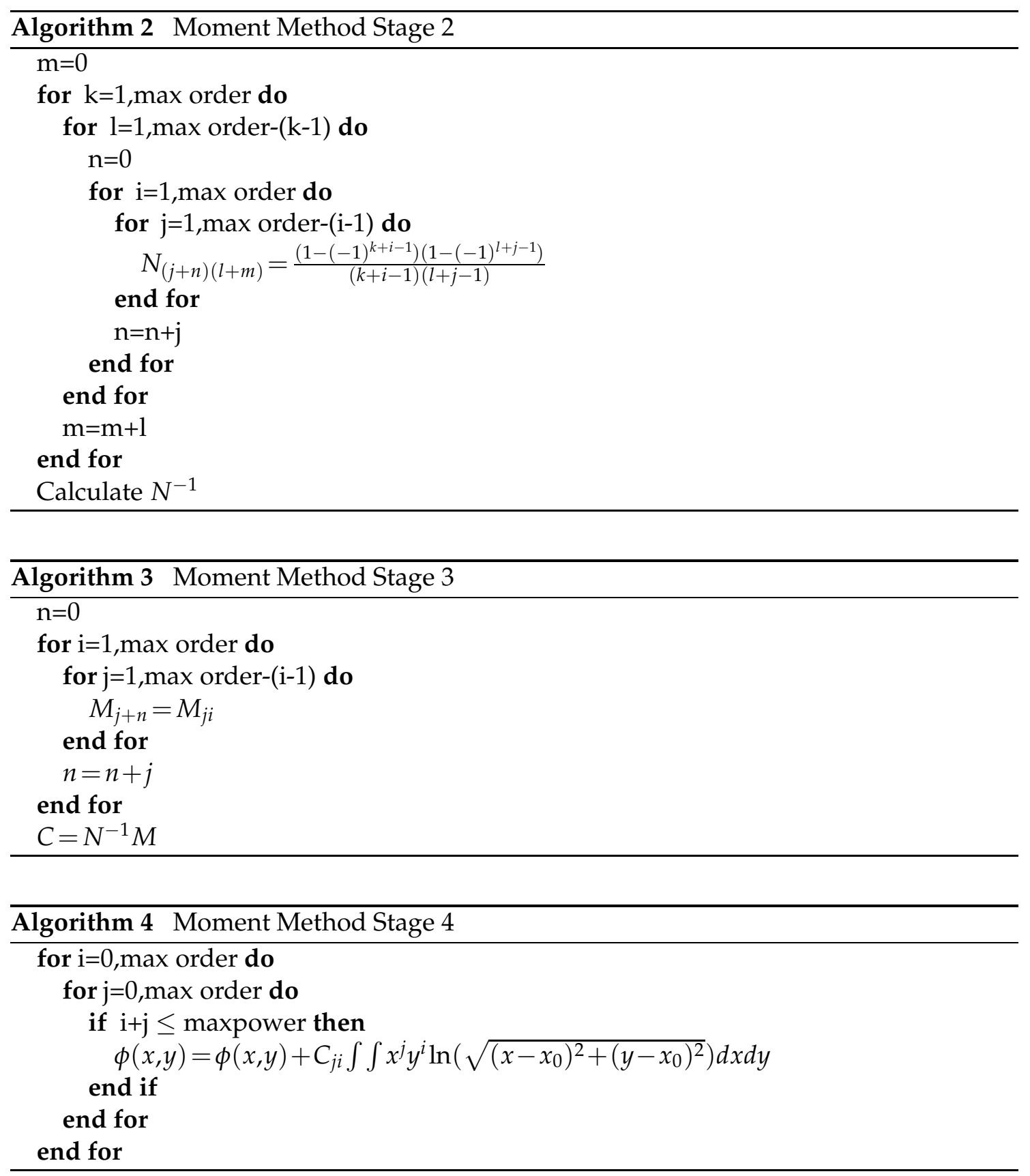

calculated integrals (see Appendix B) to create a potential function made of Differential Algebraic objects (Algorithm 4). Since the potential is its own polynomial expansion, the relevant equations of motion can be used to advance particles under the influence of the electrostatic fields of the particle distribution. 


\subsection{Using orthogonal polynomials}

The use of orthogonal polynomials is slightly different than the moment method. The corresponding steps are shown in Algorithms 5-7.

The first stage (Algorithm 5) of the orthogonal polynomial method creates a matrix of coefficients, by taking the inner product of the particles with their respective polynomials, according to (3.13). Since the distribution function is a series of delta functions, the inner product is just the polynomial with the particle as its argument.

The second stage (Algorithm 6) of this method uses the coefficients along with a Differential algebraic expansion of the orthogonal polynomials; this creates a DA object which represents the distribution function.

Finally, the coefficients in this new expansion can be used with the pre-calculated integrals from the moment method to find the potential, as shown in Algorithm 7.
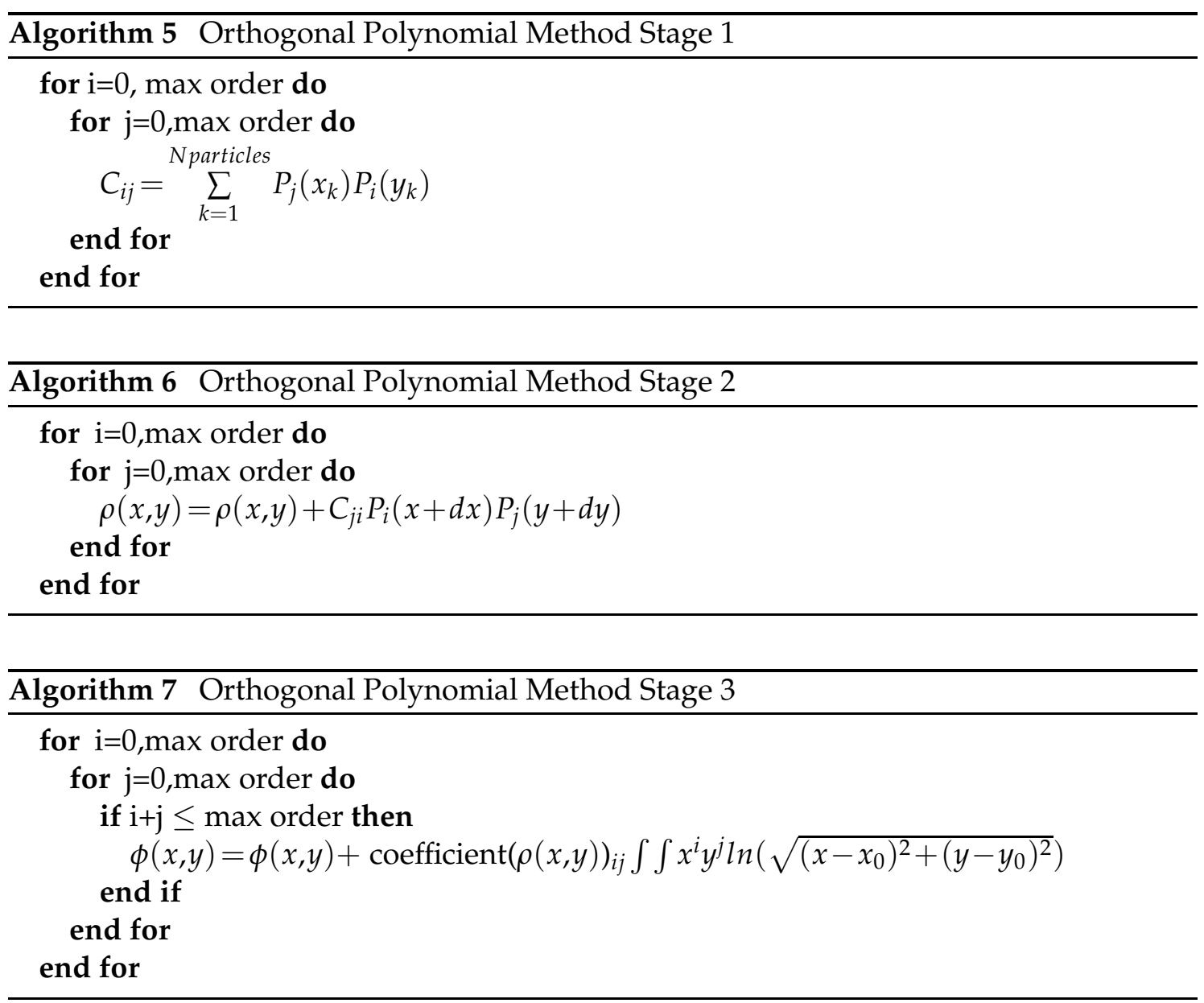


\section{Performance tests}

After the method has been implemented, it is necessary to determine how well the parts of the implementation work, and where various sources of error are. We also need to explore the best method for determining the Taylor series of the distribution function (moment or Legendre). Finally, a number of studies need to be performed in order to calibrate the use of these methods in practice. These include the number of particles for convergence of sample moments to true moments, the moment truncation order for the distribution to be well approximated, the effect of the size of the region of integration on the potential, the errors stemming from the numerical integration, and the combined effects of all these on the accuracy of the computed potential.

\subsection{Duffy transformation and differential algebras}

The first examination will look at the effectiveness of the Duffy transformation as it applies to integrating known distribution functions. This integration using differential algebras will result in a differential algebraic (DA) vector as an answer, which gives the Taylor expansion of the potential. Fig. 4 and Fig. 5 illustrate the use of the Duffy transformation to determine the three dimensional potential of a spherical and Gaussian distribution, respectively. When reading these DA vectors the coefficient column gives the coefficient of the Taylor expansion of the potential, the order column shows what order the current coefficient is, and the exponents column shows the $x, y$ and $z$ exponent values, respectively. For the case shown in Fig. 4 we can use Gauss's law to find that the electric field should be $E_{r}=(1 / 3) r$ which would give the potential as $\phi(x, y, z)=(1 / 6)\left(x^{2}+y^{2}+z^{2}\right)+(1 / 2)$ which compares very well with Fig. 4 . The differences arise both from the Runge-Kutta integrator, which has its accuracy set at $10^{-8}$, and the use of tanh to approximate the sphere; this was done to soften the edge of the distribution to make it analytic for easier numerical integration.

Next, the ways in which errors are introduced throughout the process are shown in Fig. 6. The analytical distribution which is the left most column uses a uniform square of charge integrated using the Duffy transformation. This is shown in the left most column where the only sources of error are the Runge-Kutta integrator, which in this case is set to an accuracy of $10^{-8}$ (including higher order coefficients). Next, we use the analytic distribution function to determine the moments directly from the distribution function; and compound the errors of both the moment calculation as well as the Duffy transformation, and the inversion of the matrix to find the Taylor series of the distribution; this is shown in the middle column. Finally, we sample the distribution function to create a population of test particles which follow the distribution function. The sources of error involve both the Duffy transformation, the matrix inversion, and the inherent graininess of a distribution of particles; this method is shown in the right column. 
I COEFFICIENT

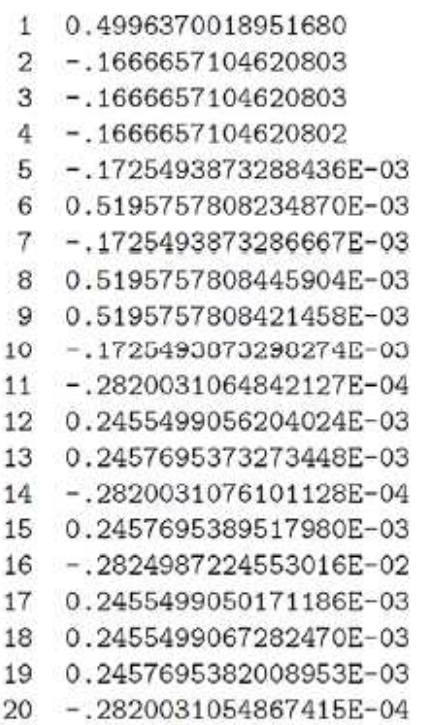

ORDER EXPONENTS

$\begin{array}{llll} & \text { x } & y & z \\ 0 & 0 & 0 & 0 \\ 2 & 2 & 0 & 0 \\ 2 & 0 & 2 & 0 \\ 2 & 0 & 0 & 2 \\ 4 & 4 & 0 & 0 \\ 4 & 2 & 2 & 0 \\ 4 & 0 & 4 & 0 \\ 4 & 2 & 0 & 2 \\ 4 & 0 & 2 & 2 \\ 4 & 0 & 0 & 4 \\ 6 & 6 & 0 & 0 \\ 6 & 4 & 2 & 0 \\ 6 & 2 & 4 & 0 \\ 6 & 0 & 6 & 0 \\ 6 & 4 & 0 & 2 \\ 6 & 2 & 2 & 2 \\ 6 & 0 & 4 & 2 \\ 6 & 2 & 0 & 4 \\ 6 & 0 & 2 & 4 \\ 6 & 0 & 0 & 6\end{array}$

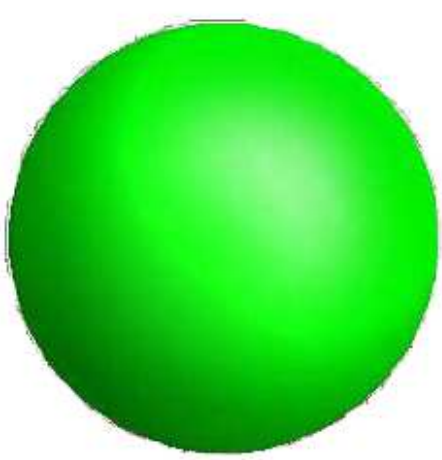

Figure 4: This shows the results of a direct DA integration of a uniform spherical distribution using the Duffy transformation. We use an analytic distribution function given by $\rho(x, y, z)=\left(1-\tanh \left(20\left(x^{2}+y^{2}+z^{2}-1\right)\right)\right) / 2$.

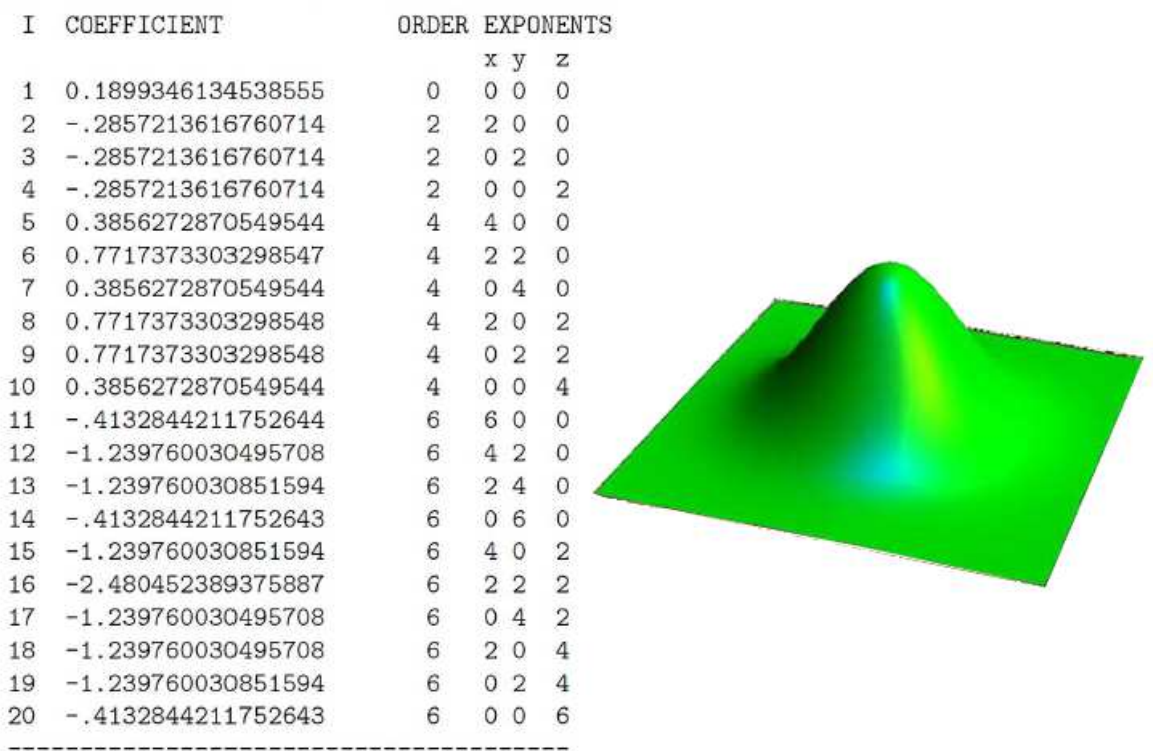

Figure 5: This shows the potential of a directly integrated Gaussian distribution in 3 dimensions using the Duffy transformation. This used the analytic distribution function $\rho(x, y, z)=\left(27 /(2 \pi)^{\frac{3}{2}}\right) \exp \left(-(9 / 2)\left(x^{2}+y^{2}+z^{2}\right)\right)$. 

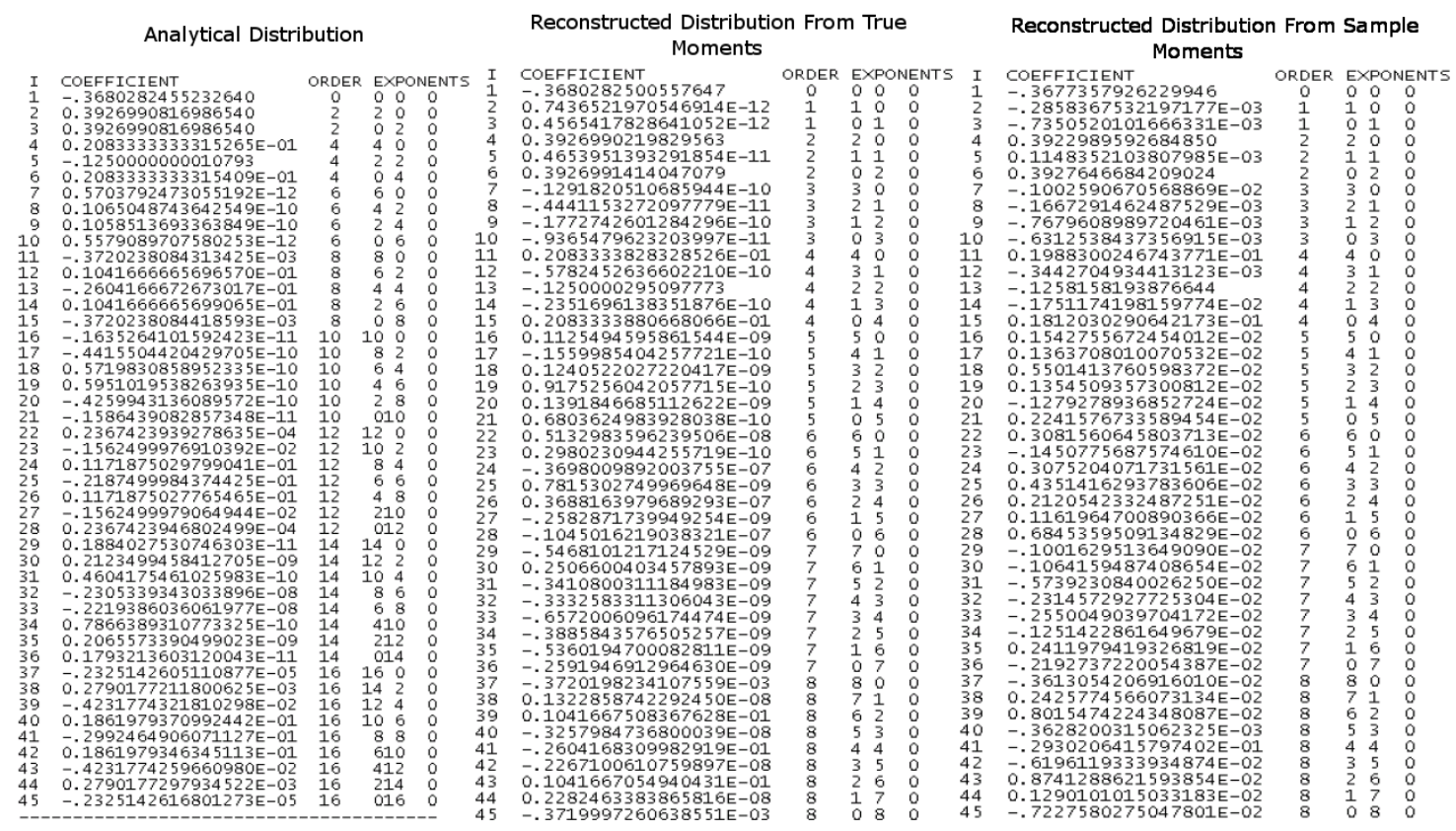

Figure 6: Comparison of various expansions of the potential of a uniform square of charge distribution. The left column shows the Duffy integration of the exact distribution. The middle column uses the moment method with the moments calculated exactly from the analytical distribution, truncated at order 16 . The column on the right shows the potential expansion obtained from sample moments using one million test particles sampled from the exact distribution function, truncated at the same order.

\subsection{Distribution reconstruction method}

The foremost question when implementing these techniques for finding the potential is which method of distribution estimation should be used. The key criteria are accuracy and speed. In order to determine which method is the best, a series of comparisons have been performed using uniform and Gaussian distributions. These methods were tested first by finding their moments through direct integration of an exactly known distribution (see Fig. 7), then by determining the moments of particle distributions derived from the exactly known distribution functions (see Fig. 8). These were then compared to potentials computed by Mathematica, using direct integration for the uniform distribution, and the Bassetti-Erskine formula for the Gaussian distribution [19], shown in (5.1),

$$
\Phi(x, y)=\int_{0}^{\infty} \frac{e^{-\frac{x^{2}}{2 \sigma_{x}^{2}+t}-\frac{y^{2}}{2 \sigma_{y}^{2}+t}}}{\sqrt{2 \sigma_{x}^{2}+t} \sqrt{2 \sigma_{y}^{2}+t}} d t .
$$

As can be seen in Fig. 7, the accuracy of the Legendre and the moment methods are roughly the same at $15^{\text {th }}$ order, but the Legendre is more accurate at $23^{\text {rd }}$ order. When we compare the results from the delta-function distributions of Fig. 8, we see the opposite effect; the moment method is now more accurate than the Legendre polynomials. The 

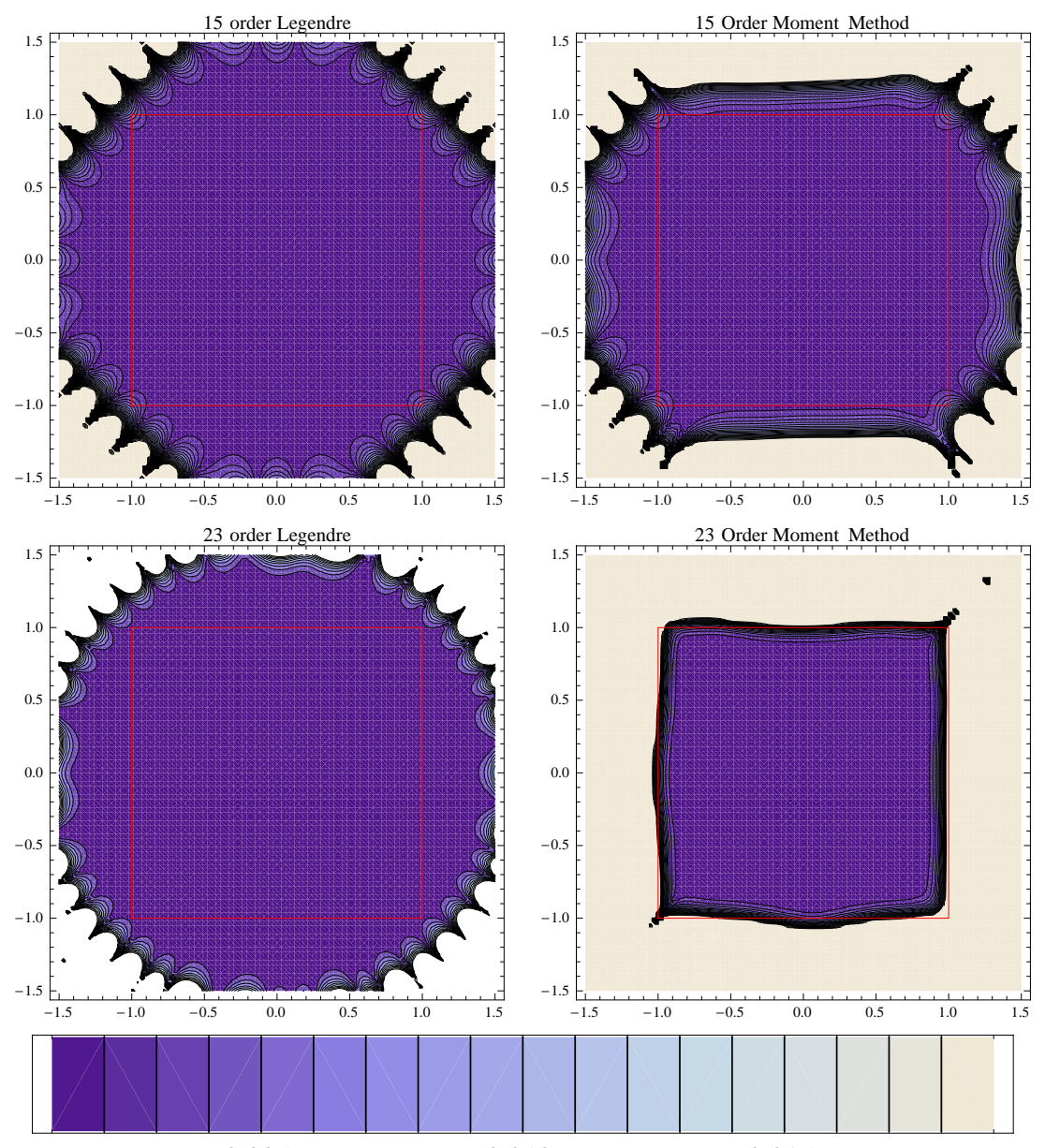

0.005

0.010

0.015

Figure 7: Comparison of the exact moment method on the right with the Legendre method on the left at $15^{\text {th }}$ and $23^{r d}$ order. These simulations used a uniform distribution with the red square denoting the region of integration. The potential was found both analytically using direct expansion around each point, and then it was determined using either the moment or Legendre methods to the indicated order, with the absolute difference indicated on the contour plot.

use of particles presents an inherent graininess to the distribution, which can be thought of as an error in the right hand side of two systems of linear equations. The error in the system is proportional to the condition number, $\kappa$, of the matrix [20],

$$
\begin{aligned}
& \vec{C}=N \vec{M}, \\
& \frac{\|\delta \vec{C}\|}{\|\vec{C}\|} \leq \kappa(N) \frac{\|\delta \vec{M}\|}{\|\vec{M}\|} .
\end{aligned}
$$

Since the moment method uses truncated SVD methods to invert its matrix, it reduces the condition number, thus making the moment method more accurate for particle systems 

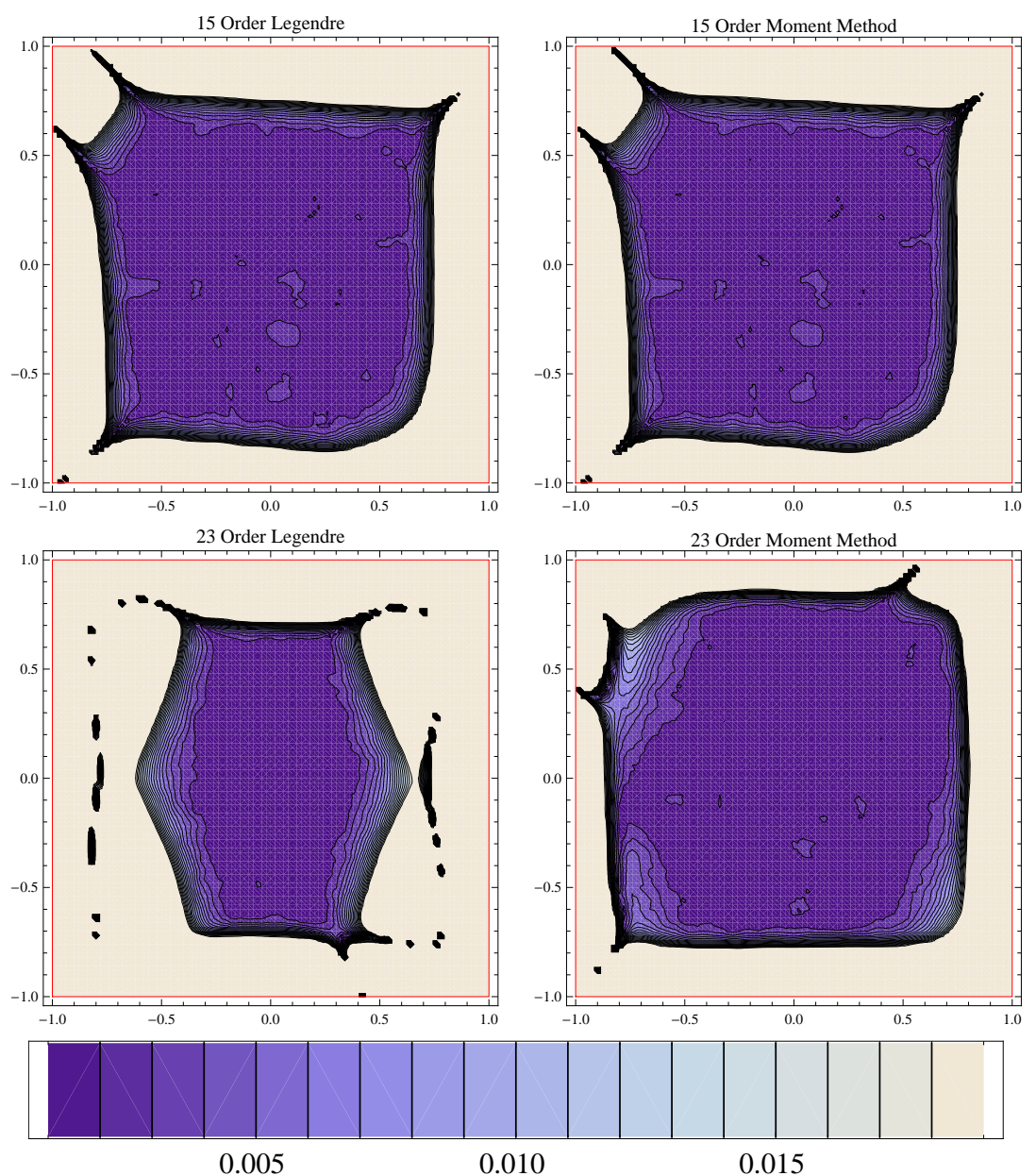

Figure 8: Comparison of the sample moment method on the right with the Legendre method on the left at $15^{\text {th }}$ and $23^{\text {rd }}$ order. These simulations used a uniform distribution of particles with one million test particles. The potential was found both analytically using direct expansion around each point, and then it was determined using either the moment or Legendre methods to the indicated order, with the absolute difference indicated on the contour plot.

at high moment orders. Combined with its higher speed, the moment method is the method of choice for the extensive simulations.

We also would like to be sure that the potentials are accurate within the bounds we wish to use for distributions that are not symmetric in the region of interest. An elliptic Gaussian beam comparison is shown in Fig. 9.

\subsection{Particle number}

The number of test particles required to get an accurate distribution must be calculated. This was done for a Gaussian distribution; some results for a couple of moments are 

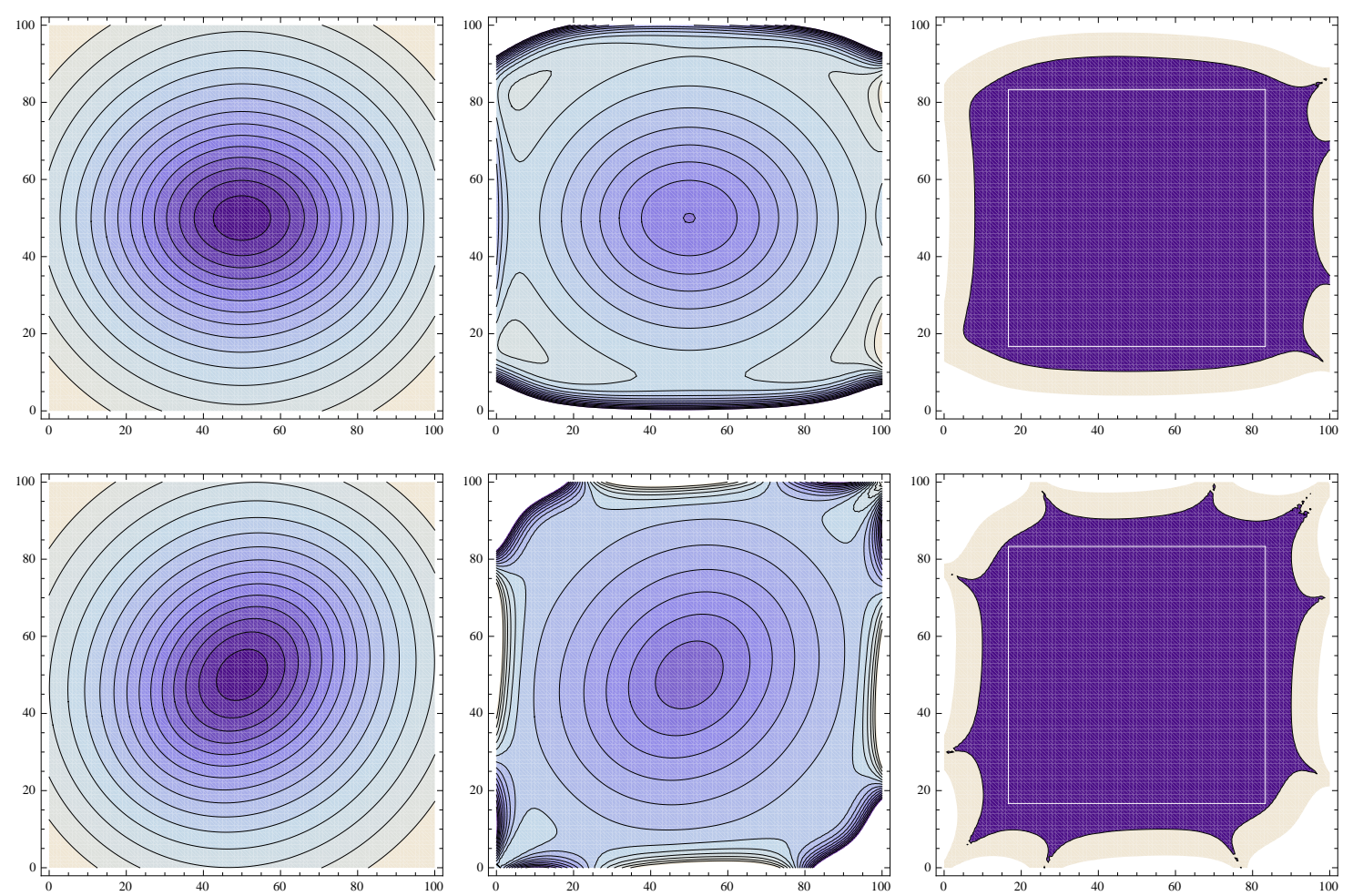

Figure 9: Comparison of two Gaussian distributions; the first showing an elliptic distribution, the second rotates the distribution by $45^{\circ}$. The left picture shows the potential as calculated by (5.1), the second is calculated using the moment method, and the third shows the absolute difference between the two with the white square denoting the region of integration. The only contour in the absolute difference column is the $1 \%$ error contour.

shown in Fig. 10. As can be seen in Fig. 10, high order moments converge slower than lower order moments. As expected, the vast majority of the elements go to zero. Overall, it is found that the number of particles should exceed 1 million for adequate convergence. This conclusion is supported by other distribution types too [11].

\subsection{Moment truncation order}

The moment truncation order is an important consideration, since the moment calculation is one of the slower parts of the method. The order at which a given accuracy is achieved should be minimized. Also, since the particle number utilized is also a factor in determining accuracy and efficiency, series convergence speeds also need to be investigated.

Accuracy was tested by first creating a set of initial conditions of uniform density with a circular cross section. The potential was computed using the exact particle-particle method of Coulomb interactions at a fixed radius at 100 equally spaced points around the center of expansion. The radius is then increased in 100 equal increments out to 1.5 times 

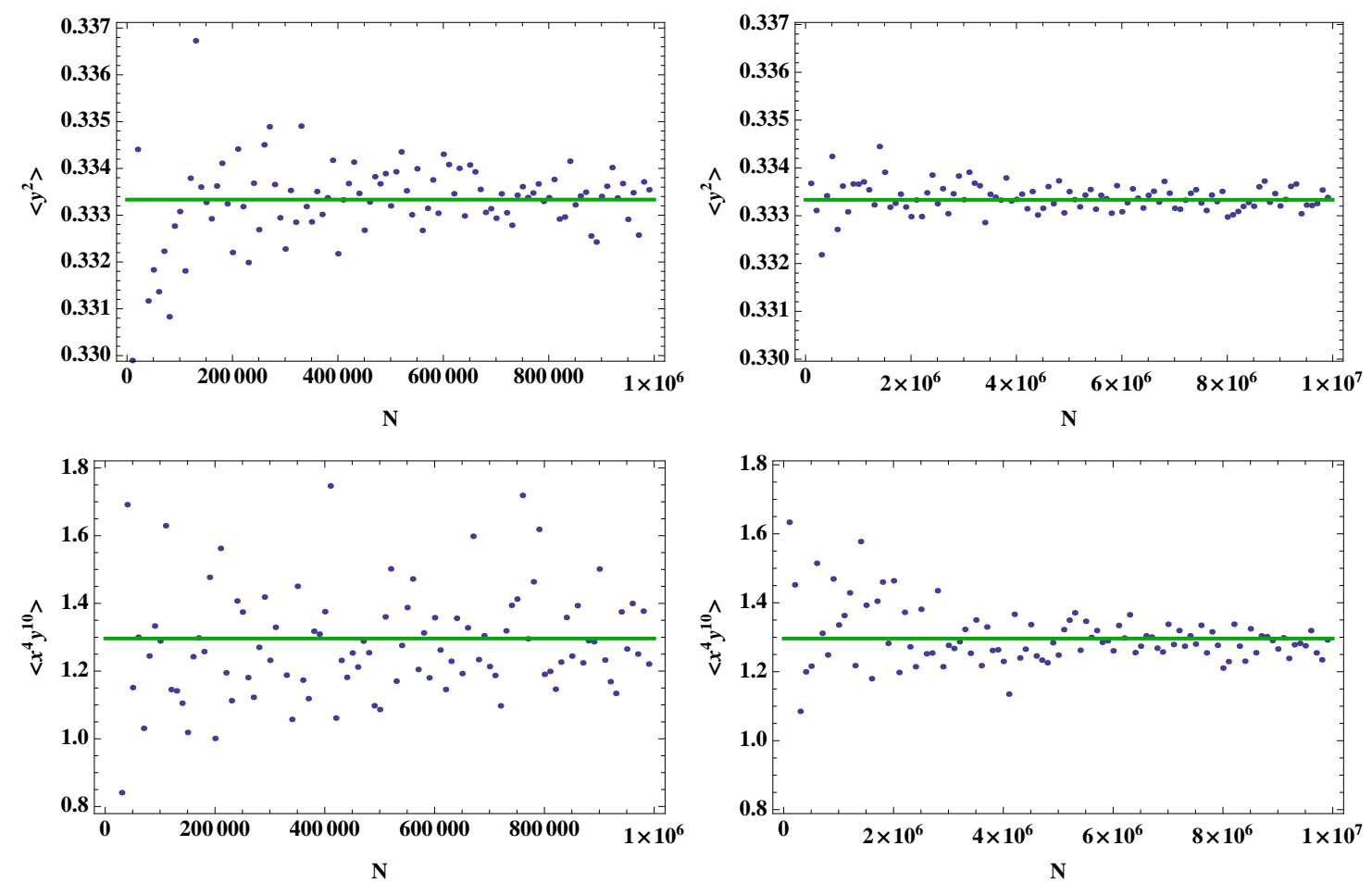

Figure 10: Comparison of a low order and a high order sample moment of a Gaussian distribution as a function of particle number with the exact values. The horizontal axis shows the particle number going up to $1 \times 10^{7}$. The difference of the plots on the left and the right is the scale of the horizontal axis. The solid lines show the exact values. Note the difference in the vertical axes scale, illustrating the difference in convergence speed. Higher order sample moments converge slower.

the size of the integration region. This process is repeated for each potential calculated using the different integration and moment orders. The integration order is the order of the Taylor series resulting from the integrals obtained by the Duffy transformations in Section 2.1. The average differences between the exact Coulomb potential and the potential calculated from the moments are summed up at the desired radius, then averaged, and used as the magnitude in Fig. 11.

As can be seen in Fig. 11, the results converge only when the integration order is larger or equal than the moment order. This follows from the fact that the values of the Taylor coefficients of the potential are dependent on the moment order, and if the moments are larger than the integration order the higher order terms will not be included. The accuracy improves with order, but when we examine the data there appears to be a drop in accuracy at high enough orders. This is caused by the ill-conditioned nature of the matrix that gets inverted and the decreased convergence speed of high order sample moments for a fixed number of particles. This gives a band of moment orders in which optimum performance is obtained. However, it is not clear if the same results hold for more complicated distributions and applications. 


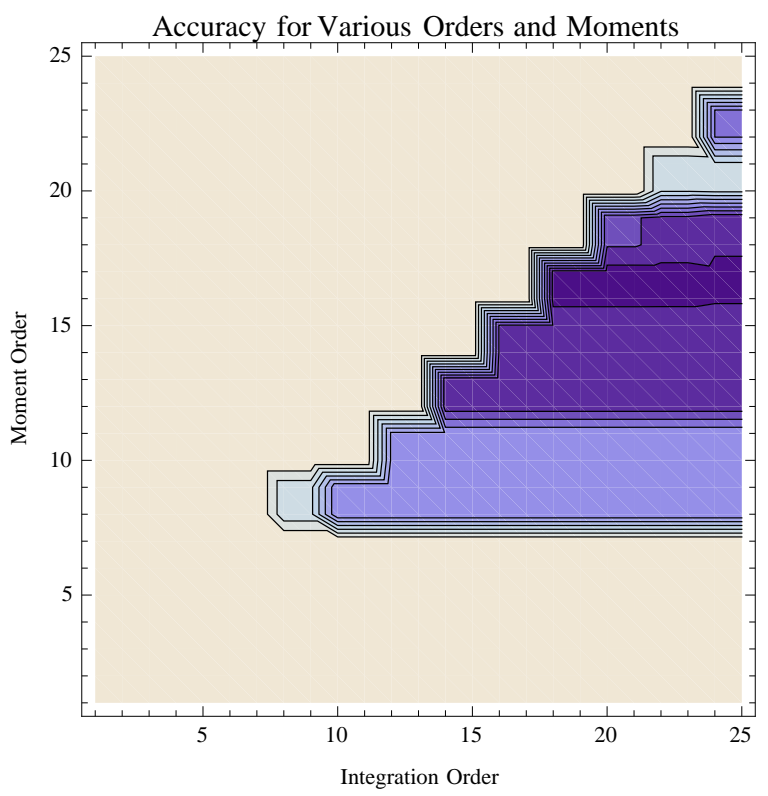

Figure 11: Contour plot of errors in the potential of a uniform circular distribution for different integration and moment orders. The darker the area the higher the accuracy. The contours show the region between 0 and 0.002 .

\subsection{Integration region}

The region over which the transformation matrix (3.9) is calculated may have a strong influence on the final answer. Since we are using a Taylor series to approximate the potential, it is necessary to choose the region carefully. The series must converge within that region fairly well so bounds must contain the points needed and not too much empty space. In distributions such as Kapchinsky-Vladimirsky where there is a definite edge the difference is not as strong, but in unbounded distributions such as a Gaussian this can cause complications. The optimal bounds will just contain "most" of the beam; in this case the support of the distribution function just barely fits into the integration region, and simultaneously the resulting polynomial approximation of the distribution function will result in optimal accuracy over that region at a fixed order of the polynomial. In the case of Gaussian, an empirical test shows that the optimal truncation is at a distance of around $5 \sigma$ from the center [24].

\subsection{Beam propagation example}

One method of validating the methods of this paper involves comparing the results from this method to an example that has a known analytic solution. The example provided involved tracking a $100 \mathrm{KeV}$ laminar electron beam with an initial radius of $r_{0}=5 \mathrm{~mm}$ and a current of $I=1 \mathrm{~A}$. This beam was propagated over a distance of $z=20 \mathrm{~cm}$ and approximated using $10^{6}$ test particles. This dynamics problem has an analytic solution 


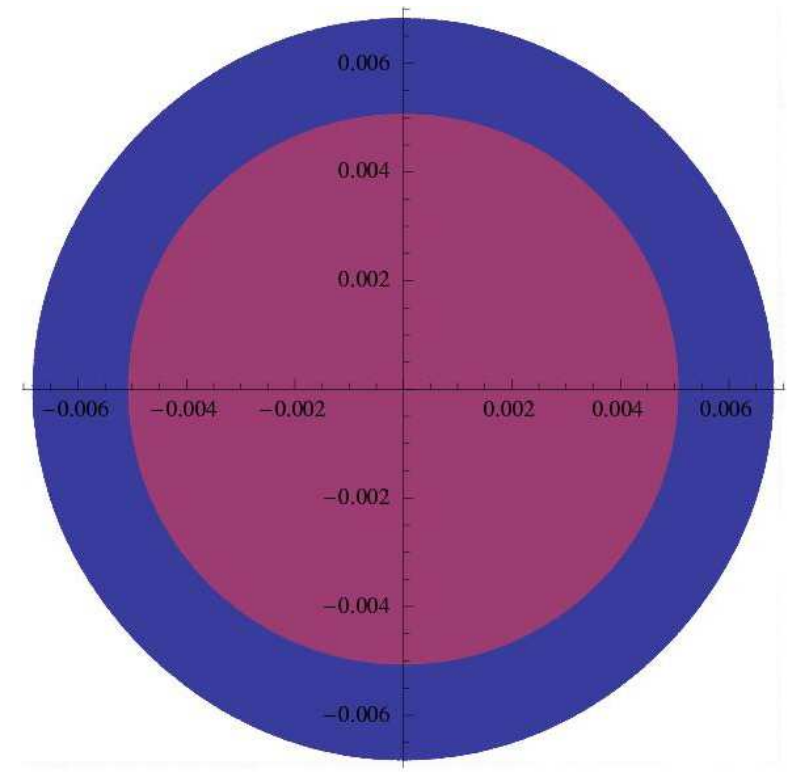

Figure 12: A comparison of the initial and final horizontal and vertical test particle positions in a $20 \mathrm{~cm}$ drift at $100 \mathrm{KeV}$ beam energy $1 \mathrm{~A}$ of current with an initial radius of $5 \mathrm{~mm}$. Purple indicates the initial points, blue the final.

for the ratio of final to initial beam sizes given by [21],

$$
\frac{r_{m}}{r_{0}}=1+5.87 \times 10^{-5} \frac{I}{\left(\gamma^{2}-1\right)^{\frac{3}{2}}}\left(\frac{z}{r_{0}}\right)^{2},
$$

which predicts a beam size growth of $33 \%$. In this equation $I$ is the beam current, $\gamma$ is the relativistic factor, $r_{m}$ and $r_{0}$ are the final and initial radii respectively, and $z$ is the propagation distance. Two methods employed involved placing test particles on the edge, and comparing their initial and final positions as well as examining the linear dependence of the final position of a given particle on its initial position, which we refer to as $\left(x_{f} \mid x_{i}\right)$, $\left(y_{f} \mid y_{i}\right)$ for the horizontal and vertical dependence, respectively. A comparison of the initial to final distribution can be seen in Fig. 12, and a comparison of the final results can be seen in Table 1. Therefore, the two methods bracket the analytic solution with relative error less than $2 \%$, which is within the accuracy of the analytical result (5.4).

Table 1: Table of different methods for finding the increase in size from beginning to end in the $20 \mathrm{~cm}$ drift at $100 \mathrm{KeV}$ beam energy $1 \mathrm{~A}$ of current with an initial radius of $5 \mathrm{~mm}$.

\begin{tabular}{||c|c||}
\hline Method & Growth \\
\hline Edge Point $\mathrm{x}$ & $35.27 \%$ \\
Edge Point $\mathrm{y}$ & $35.30 \%$ \\
$\left(x_{f} \mid x_{i}\right)$ & $31.21 \%$ \\
$\left(y_{f} \mid y_{i}\right)$ & $31.34 \%$ \\
\hline
\end{tabular}




\section{Summary and conclusions}

In this paper we presented a Poisson solver that uses differential algebraic techniques to convert diverse distributions into Taylor series expansions of the potential. The method can be used to convert a set of test particles into a Taylor series distribution using either the statistical properties of the particles, or by projecting the test particles onto a set of orthogonal polynomials. The resulting set of coefficients of the Taylor series can then be multiplied by the pre-computed integrals of a generic Taylor series to find the potential.

The pre-computed integrals are calculated using a generic polynomial series and a dimensionally appropriate Green's function. In order to avoid numerical singularities arising from the Green's function a Duffy transformation is used to remove the singularities. The differential algebras used in these integrals not only calculate the potential at the center of the distribution, but also the Taylor series expansion around that point. The combination of these individual expansions with the coefficients calculated from the distribution provides an expansion of the potential within the region of interest and sometimes beyond.

We found that for maximum accuracy at least $1 \times 10^{6}$ particles were needed, that the region of integration needs to be a square that encompasses the distribution without being too much larger, and that this method could model distributions that were not entirely symmetric. We also determined that the use of the statistical moments was superior in accuracy to the use of orthogonal polynomials since the method used to convert the moments of a point distribution is more resilient to problems caused by the graininess of a point distribution. We found that, when using the moment method, the ideal moment order was 16 with the order of integration for the pre-computed integrals being greater than or equal to 16 for near-uniform distributions.

Finally, there are (multi-modal, oscillatory) distributions that are not easily modeled accurately with $16^{\text {th }}$ order Taylor series. For these cases the methods of this paper still allow the extraction of potential and electric field maps, but their accuracy will be limited. While they may not be accurate enough for propagation of the charge distribution, it still might be useful for local analysis purposes. The push of particles in this case can be solved with one of the various other existing methods such as the fast multipole method. This way one can determine and analyze the dynamics of the central core of a beam. Exploration of these ideas is the subject of ongoing work.

\section{Acknowledgments}

This work was supported by the Department of Energy under contract DE-FG02-08ER41532 with Northern Illinois University. 


\section{A DA vectors and their uses}

Differential Algebraic (DA) methods are mathematical constructs that allow high order derivatives computation numerically without truncation error [22]. This can the thought of as generalizations of computational differentiation [23], and it is a much larger field of study. The area we are interested in relies on computation of Taylor expansions of flows of ordinary differential equations, and the ability of differential algebraic methods to determine very high order derivatives of multivariable functions very accurately. Here we are enumerating a few examples that in some way are used in the body of the paper; we mostly follow the exposition of [13].

In the simplest case, a first order one variable structure ${ }_{1} D_{1}$, with its elements being an ordered set of two real numbers $\left(q_{0}, q_{1}\right)$, is defined by the following relations:

$$
\begin{aligned}
& \left(q_{0}, q_{1}\right)+\left(r_{0}, r_{1}\right)=\left(q_{0}+r_{0}, q_{1}+r_{1}\right), \\
& t\left(q_{0}, q_{1}\right)=\left(t q_{0}, t q_{1}\right), \\
& \left(q_{0}, q_{1}\right) \cdot\left(r_{0}, r_{1}\right)=\left(q_{0} r_{0}, q_{0} r_{1}+q_{1} r_{0}\right), \\
& \left(q_{0}, q_{1}\right)^{n}=\left(q_{0}^{n}, n q_{0}^{n-1} q_{1}\right),
\end{aligned}
$$

where $t$ is a scalar and $n$ is an integer.

\section{A.1 Computation of derivatives}

Assuming we are using the ${ }_{1} D_{1}$ structure, the DA vector $x$ is shown as $\left(x_{0}, x_{1}\right)$. In this example we have the equation $f$ and its derivative,

$$
\begin{aligned}
& f(x)=x^{3}+3 x-\frac{2}{x+1} \\
& f^{\prime}(x)=3 x^{2}+3+\frac{2}{(x+1)^{2}}
\end{aligned}
$$

which at $x=3$ gives

$$
\begin{aligned}
& f(3)=35 \frac{1}{2} \\
& f^{\prime}(3)=30 \frac{1}{8}
\end{aligned}
$$

If instead we evaluate the function replacing $x$ with $(3,1)$ we will get,

$$
f((3,1))=((3,1))^{3}+3(3,1)-\frac{2}{(3,1)+1}
$$




$$
\begin{aligned}
& f((3,1))=\left(27,3(3)^{2} 1\right)+(9,3)-\frac{2}{(4,1)}, \\
& f((3,1))=(27,27)+(9,3)-2\left(\frac{1}{4}, \frac{-1}{16}\right), \\
& f((3,1))=\left(35 \frac{1}{2}, 30 \frac{1}{8}\right) .
\end{aligned}
$$

Thus, the first entry (or the constant part) in the vector is the value the function takes at that particular point, and the second entry is the derivative at the same point. For higher orders and larger variable numbers the vectors become longer and more complicated, but conceptually the same procedure is followed. The vector eventually takes the form of essentially a high order multivariate Taylor series of the function $f$.

\section{A.2 Integration}

An antiderivative can be quickly determined through simple polynomial integration of the DA vector. Assuming a vector $q$,

$$
q=\left(q_{0}, q_{1}, q_{2}, q_{3}, \cdots\right)
$$

its integral $Q$ can be determined simply through a coefficient change,

$$
Q=\left(c, q_{0}, \frac{q_{1}}{2}, \frac{q_{2}}{3}, \frac{q_{3}}{4}, \cdots\right),
$$

where $c$ is an arbitrary constant. Furthermore, since DA vectors work by redefining mathematical relations, other basic programs like a Runge-Kutta integrator will carry the DA vectors through, this would allow in our case for a Taylor series of the potential for an entire region to be calculated using only an integration of a reference particle.

Another way that this can be used is in the case of a function such as $\sin (x)$. It can be calculated as $\sin (x+d x)$ in DA. On the other hand, $\sin (x+d x)$ can also be rewritten as $\sin (x)+\sin ^{\prime}(x) d x$, or $\sin (x)+\cos (x) d x$. This is shown as an example of the resultant DA operations in the vector in Fig. 13.

\section{A.3 DA-ODE integration}

One method that has been a tremendous speed boost in this work is the use of DA methods to solve ordinary differential equations (ODEs). This method uses DA integration to solve a system of first order ODEs. The method works in cases with no explicit time dependence, since any first order ODE of this type can be written as,

$$
\frac{d \vec{z}}{d s}=\vec{F}(\vec{z})
$$

$\vec{z}\left(s_{i}\right)=\vec{z}_{i}$. 


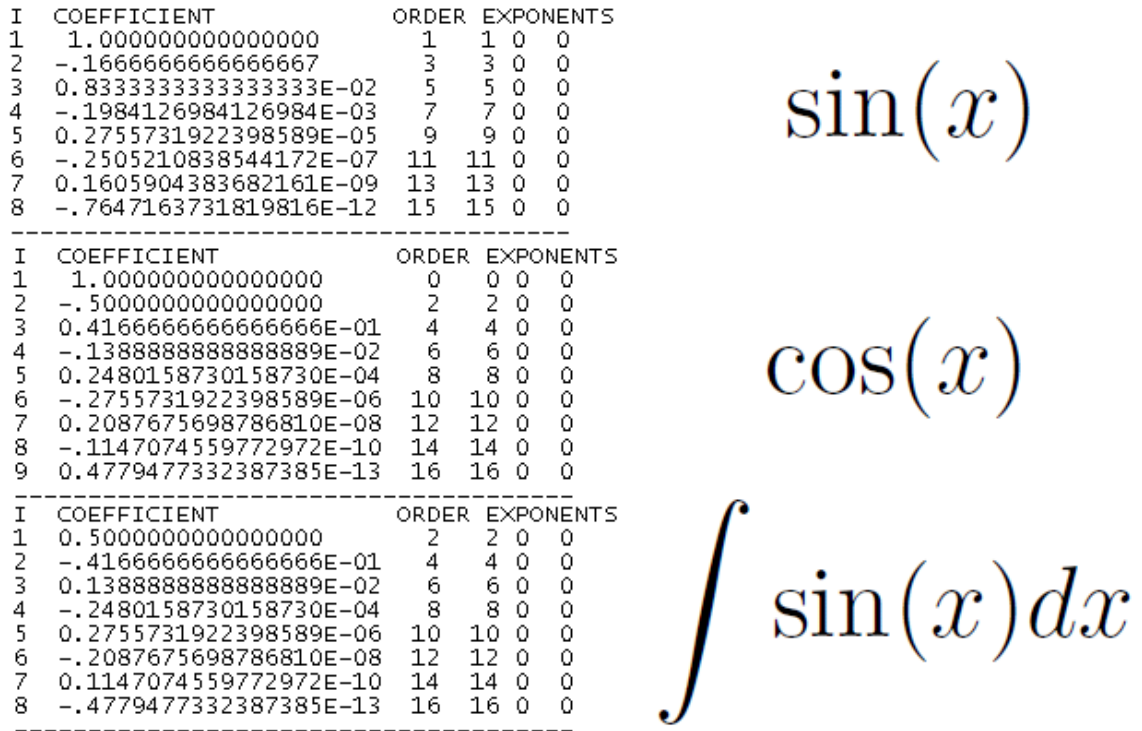

Figure 13: This shows examples of $16^{\text {th }}$ order one dimensional DA vectors for various functions. The expansions are around $x=0$.

This equation is then recast as an integral equation,

$$
\vec{z}\left(s_{f}\right)=\vec{z}_{i}+\int_{s_{i}}^{s_{f}} \vec{F}(\vec{z}) d s,
$$

and solved DA-integration as shown above in Subsection A.2. This method is also employed in computing the Duffy-transformed integrals by recasting the integrals as initial value problems and solving the associated ODEs.

\section{B Scaling to improve efficiency}

There are a number of numerical concerns when working with high order polynomials on computers. If the bounds of integration are chosen improperly the numbers that result can become unusably large or so small that the computer truncates them to zero. Therefore, when determining the distribution function, we have chosen to cast the test particles in a coordinate system that corresponds to a size scale that provides optimal numerical stability. Thus, rather than SI units, the Taylor coefficients are determined in units such that the distribution spans a square that goes from $(-1,1)$ on each side.

The scaling of the integrals is given using dimensional analysis. If the distributions are given as,

$$
\begin{aligned}
& \rho_{\text {a.u. }}\left(x_{\text {a.u., }} y_{\text {a.u. }}\right)=C_{00}+C_{10} x_{\text {a.u. }}+C_{01} y_{\text {a.u. }}+\cdots, \\
& \rho_{\text {S.I. }}\left(x_{\text {S.I. }} y_{\text {S.I. }}\right)=D_{00}+D_{10} x_{\text {S.I. }}+C_{01} y_{\text {S.I. }}+\cdots,
\end{aligned}
$$


the coefficients are going to have a form $C_{n m}=[[\rho]] / \ell_{a . u .}^{n+m+2}$ and $D_{n m}=[[\rho]] / \ell_{\text {S.I. }}^{n+m+2}$. Then, assuming that $x_{\text {S.I. }}=a x_{a . u}$. and $y_{\text {S.I. }}=a y_{a . u}$. with the same scaling factor $a$, the potentials will be given as

$$
\begin{aligned}
\phi_{a . u .}\left(x_{0, a . u .}, y_{0, a . u .}\right)= & \sum_{n, m} C_{n m} \int_{-1}^{1} \int_{-1}^{1} x_{a . u .}^{n} y_{a . u .}^{m} \\
& \times \ln \left(\sqrt{\left(x_{a . u .}-x_{0, a . u .}\right)^{2}+\left(y_{a . u .}-y_{0, a . u .}\right)^{2}}\right) d x_{a . u .} d y_{a . u . \prime} \\
\phi_{\text {S.I. }}\left(x_{0, \text { S.I. }}, y_{0, S . I .}\right)= & \sum_{n, m} D_{n m} \int_{-a}^{a} \int_{-a}^{a} x_{\text {S.I. }}^{n} y_{\text {S.I. }}^{m} \\
& \times \ln \left(\sqrt{\left(x_{\text {S.I. }}-x_{0, \text { S.I. }}\right)^{2}+\left(y_{\text {S.I. }}-y_{0, S . I .}\right)^{2}}\right) d x_{\text {S.I. }} d y_{\text {S.I. }} .
\end{aligned}
$$

The integral can be scaled in the following way,

$$
\begin{aligned}
& I_{n m, S . I .}\left(x_{0}, y_{0}\right)=\int_{-1}^{1} \int_{-1}^{1}\left(a x_{a . u .}\right)^{n}\left(a y_{a . u .}\right)^{m} \\
& \times \ln \left(\sqrt{\left(a x_{a . u .}-a x_{0, a . u .}\right)^{2}+\left(a y_{a . u .}-a y_{0, a . u .}\right)^{2}}\right) a d x_{a . u .} a d y_{a . u . \prime} \\
& I_{n m, S . I .}\left(x_{0}, y_{0}\right)=a^{n+m+2} \int_{-1}^{1} \int_{-1}^{1} x_{a . u .}^{n} y_{a . u .}^{m} \ln (a) \\
& +x_{\text {a.u. }}^{n} y_{\text {a.u. }}^{m} \ln \left(\sqrt{\left(x_{\text {a.u. }}-x_{0, a . u .}\right)^{2}+\left(y_{a . u .}-y_{0, a . u .}\right)^{2}}\right) d x_{\text {a.u. }} d y_{a . u .} \\
& I_{n m, \text { S.I. }}\left(x_{0}, y_{0}\right)=a^{n+m+2}\left(\frac{\left(1-(-1)^{n+1}\right)\left(1-(-1)^{m+1}\right)}{(n+1)(m+1)} \ln (a)\right. \\
& \left.+\int_{-1}^{1} \int_{-1}^{1} x_{a . u .}^{n} y_{a . u .}^{m} \ln \left(\sqrt{\left(x_{a . u .}-x_{0, a . u .}\right)^{2}+\left(y_{a . u .}-y_{0, a . u .}\right)^{2}}\right) d x_{a . u .} d y_{a . u .}\right), \\
& I_{n m, S . I .}\left(x_{0}, y_{0}\right)=a^{n+m+2}\left[\frac{\left(1-(-1)^{n+1}\right)\left(1-(-1)^{m+1}\right)}{(n+1)(m+1)} \ln (a)+I_{n m, a . u .}\left(\frac{x_{0}}{a}, \frac{y_{0}}{a}\right)\right] .
\end{aligned}
$$

Combined with the scaling of the coefficients, the potential becomes

$$
\phi_{\text {S.I. }}\left(x_{0}, y_{0}\right)=\sum_{n m} C_{n m}\left(\frac{\left(1-(-1)^{n+1}\right)\left(1-(-1)^{m+1}\right)}{(n+1)(m+1)} \ln (a)+I_{n m, a . u .}\left(\frac{x_{0}}{a}, \frac{y_{0}}{a}\right)\right),
$$

where $I_{n m, a . u .}(x, y)$ have been pre-computed and stored. 


\section{3D Duffy transformation}

In this instance we would like to find the integral over the parallelepiped,

$$
I=\int_{a}^{b} \int_{c}^{d} \int_{e}^{f} \frac{\rho(x, y, z)}{\sqrt{\left(x-x_{0}\right)^{2}+\left(y-y_{0}\right)^{2}+\left(z-z_{0}\right)^{2}}} d x d y d z
$$

If we refer to the integrand as $\Xi$, we can cut the cube into eight smaller cubes and rearrange their bounds of integration such that we get

$$
\begin{aligned}
\int_{a}^{b} \int_{c}^{d} \int_{e}^{f} \Xi d x d y d z= & -\int_{z_{0}}^{e} \int_{x_{0}}^{a} \int_{y_{0}}^{c} \Xi d x d y d z+\int_{z_{0}}^{e} \int_{x_{0}}^{a} \int_{y_{0}}^{d} \Xi d x d y d z \\
& +\int_{z_{0}}^{e} \int_{x_{0}}^{b} \int_{y_{0}}^{c} \Xi d x d y d z-\int_{z_{0}}^{e} \int_{x_{0}}^{b} \int_{y_{0}}^{d} \Xi d x d y d z \\
& +\int_{z_{0}}^{f} \int_{x_{0}}^{a} \int_{y_{0}}^{c} \Xi d x d y d z-\int_{z_{0}}^{f} \int_{x_{0}}^{a} \int_{y_{0}}^{d} \Xi d x d y d z \\
& -\int_{z_{0}}^{f} \int_{x_{0}}^{b} \int_{y_{0}}^{c} \Xi d x d y d z+\int_{z_{0}}^{f} \int_{x_{0}}^{b} \int_{y_{0}}^{d} \Xi d x d y d z .
\end{aligned}
$$

Similarly to the 2D case, we focus on one of the integrals, namely

$$
\int_{z_{0}}^{f} \int_{x_{0}}^{b} \int_{y_{0}}^{d} \frac{\rho(x, y, z) d x d y d z}{\sqrt{\left(x-x_{0}\right)^{2}+\left(y-y_{0}\right)^{2}+\left(z-z_{0}\right)^{2}}}
$$

which we rescale to a unit cube by means of the coordinate transformation,

$$
\begin{aligned}
& u_{1}=\frac{x-x_{0}}{b-x_{0}}, \\
& u_{2}=\frac{y-y_{0}}{d-y_{0}}, \\
& u_{3}=\frac{z-z_{0}}{f-z_{0}} .
\end{aligned}
$$

Again, for convenience we set $\lambda_{1}=\left(b-x_{0}\right), \lambda_{2}=\left(d-y_{0}\right)$, and $\lambda_{3}=\left(f-z_{0}\right)$. This gives the differential

$$
d x d y d z=\lambda_{1} \lambda_{2} \lambda_{3} d u_{1} d u_{2} d u_{3}
$$

The integral then becomes

$$
I=\lambda_{1} \lambda_{2} \lambda_{3} \int_{0}^{1} \int_{0}^{1} \int_{0}^{1} \frac{\rho\left(\lambda_{1} u_{1}+x_{0}, \lambda_{2} u_{2}+y_{0}, \lambda_{3} u_{3}+z_{0}\right)}{\sqrt{\lambda_{1}^{2} u_{1}^{2}+\lambda_{2}^{2} u_{2}^{2}+\lambda_{3}^{2} u_{3}^{2}}} d u_{1} d u_{2} d u_{3} .
$$


The integral is now a unit cube, which we map into three pyramids the same way we mapped the square into two triangles in the section describing the $2 \mathrm{D}$ procedure. This is done by rewriting the integral to read

$$
\begin{aligned}
I= & \lambda_{1} \lambda_{2} \lambda_{3} \int_{0}^{1} \int_{0}^{u_{1}} \int_{0}^{u_{1}} \frac{\rho\left(\lambda_{1} u_{1}+x_{0}, \lambda_{2} u_{2}+y_{0}, \lambda_{3} u_{3}+z_{0}\right)}{\sqrt{\lambda_{1}^{2} u_{1}^{2}+\lambda_{2}^{2} u_{2}^{2}+\lambda_{3}^{2} u_{3}^{2}}} d u_{1} d u_{2} d u_{3} \\
& +\lambda_{1} \lambda_{2} \lambda_{3} \int_{0}^{1} \int_{0}^{u_{2}} \int_{0}^{u_{2}} \frac{\rho\left(\lambda_{1} u_{1}+x_{0}, \lambda_{2} u_{2}+y_{0}, \lambda_{3} u_{3}+z_{0}\right)}{\sqrt{\lambda_{1}^{2} u_{1}^{2}+\lambda_{2}^{2} u_{2}^{2}+\lambda_{3}^{2} u_{3}^{2}}} d u_{2} d u_{3} d u_{1} \\
& +\lambda_{1} \lambda_{2} \lambda_{3} \int_{0}^{1} \int_{0}^{u_{3}} \int_{0}^{u_{3}} \frac{\rho\left(\lambda_{1} u_{1}+x_{0}, \lambda_{2} u_{2}+y_{0}, \lambda_{3} u_{3}+z_{0}\right)}{\sqrt{\lambda_{1}^{2} u_{1}^{2}+\lambda_{2}^{2} u_{2}^{2}+\lambda_{3}^{2} u_{3}^{2}}} d u_{3} d u_{1} d u_{2} .
\end{aligned}
$$

Each integral then has its pyramid rescaled into a unit cube; for the first integral,

$$
\begin{array}{lll}
w_{1}=u_{1}, & w_{2}=\frac{u_{2}}{u_{1}}, & w_{3}=\frac{u_{3}}{u_{1}}, \\
u_{1}=w_{1}, & u_{2}=w_{1} w_{2}, & u_{3}=w_{1} w_{3},
\end{array}
$$

for the second integral,

$$
\begin{array}{lll}
w_{1}=\frac{u_{1}}{u_{2}}, & w_{2}=u_{2}, & w_{3}=\frac{u_{3}}{u_{2}}, \\
u_{1}=w_{1} w_{2}, & u_{2}=w_{2}, & u_{3}=w_{2} w_{3},
\end{array}
$$

and for the third,

$$
\begin{array}{lll}
w_{1}=\frac{u_{1}}{u_{3}}, & w_{2}=\frac{u_{2}}{u_{3}}, & w_{3}=u_{3}, \\
u_{1}=w_{1} w_{3}, & u_{2}=w_{3} w_{2}, & u_{3}=w_{3} .
\end{array}
$$

The integral now becomes

$$
\begin{aligned}
I= & \lambda_{1} \lambda_{2} \lambda_{3} \int_{0}^{1} \int_{0}^{1} \int_{0}^{1} \frac{w_{1}^{2} \rho\left(\lambda_{1} w_{1}+x_{0}, \lambda_{2} w_{1} w_{2}+y_{0}, \lambda_{3} w_{1} w_{3}+z_{0}\right)}{\sqrt{\lambda_{1}^{2} w_{1}^{2}+\lambda_{2}^{2} w_{1}^{2} w_{2}^{2}+\lambda_{3}^{2} w_{1}^{2} w_{3}^{2}}} d w_{1} d w_{2} d w_{3} \\
& +\lambda_{1} \lambda_{2} \lambda_{3} \int_{0}^{1} \int_{0}^{1} \int_{0}^{1} \frac{w_{2}^{2} \rho\left(\lambda_{1} w_{2} w_{1}+x_{0}, \lambda_{2} w_{2}+y_{0}, \lambda_{3} w_{2} w_{3}+z_{0}\right)}{\sqrt{\lambda_{1}^{2} w_{2}^{2} w_{1}^{2}+\lambda_{2}^{2} w_{2}^{2}+\lambda_{3}^{2} w_{2}^{2} w_{3}^{2}}} d w_{1} d w_{2} d w_{3} \\
& +\lambda_{1} \lambda_{2} \lambda_{3} \int_{0}^{1} \int_{0}^{1} \int_{0}^{1} \frac{w_{3}^{2} \rho\left(\lambda_{1} w_{3} w_{1}+x_{0}, \lambda_{2} w_{3} w_{2}+y_{0}, \lambda_{3} w_{3}+z_{0}\right)}{\sqrt{\lambda_{1}^{2} w_{3}^{2} w_{1}^{2}+\lambda_{2}^{2} w_{3}^{2} w_{2}^{2}+\lambda_{3}^{2} w_{3}^{2}}} d w_{1} d w_{2} d w_{3}
\end{aligned}
$$


which can be simplified to

$$
\begin{aligned}
I= & \lambda_{1} \lambda_{2} \lambda_{3} \int_{0}^{1} \int_{0}^{1} \int_{0}^{1} \frac{w_{1} \rho\left(\lambda_{1} w_{1}+x_{0}, \lambda_{2} w_{1} w_{2}+y_{0}, \lambda_{3} w_{1} w_{3}+z_{0}\right)}{\sqrt{\lambda_{1}^{2}+\lambda_{2}^{2} w_{2}^{2}+\lambda_{3}^{2} w_{3}^{2}}} d w_{1} d w_{2} d w_{3} \\
& +\lambda_{1} \lambda_{2} \lambda_{3} \int_{0}^{1} \int_{0}^{1} \int_{0}^{1} \frac{w_{2} \rho\left(\lambda_{1} w_{2} w_{1}+x_{0}, \lambda_{2} w_{2}+y_{0}, \lambda_{3} w_{2} w_{3}+z_{0}\right)}{\sqrt{\lambda_{1}^{2} w_{1}^{2}+\lambda_{2}^{2}+\lambda_{3}^{2} w_{3}^{2}}} d w_{1} d w_{2} d w_{3} \\
& +\lambda_{1} \lambda_{2} \lambda_{3} \int_{0}^{1} \int_{0}^{1} \int_{0}^{1} \frac{w_{3} \rho\left(\lambda_{1} w_{3} w_{1}+x_{0}, \lambda_{2} w_{3} w_{2}+y_{0}, \lambda_{3} w_{3}+z_{0}\right)}{\sqrt{\lambda_{1}^{2} w_{1}^{2}+\lambda_{2}^{2} w_{2}^{2}+\lambda_{3}^{2}}} d w_{1} d w_{2} d w_{3} .
\end{aligned}
$$

Again, the singularity is now removed and the integrals can be computed using the eighth order Runge-Kutta routine using adaptive step size.

\section{References}

[1] W. Henning, C. Shank (Eds.), Accelerators for America's Future, Department of Energy, 2010.

[2] A. W. Chao, W. Chou (Eds.), Reviews of Accelerator Science and Technology, World Scientific Publishing Co. Pte. Ltd., Singapore, 2008.

[3] J. M. Dawson, Particle simulation of plasmas, Rev. Mod. Phys. 55 (2) (1983) 403-447.

[4] R. W. Hockney, J. W. Eastwood, Computer Simulation Using Particles, McGraw-Hill, New York, 1981.

[5] B. Terzic, I. Pogorelov, C. Bohn, Particle-in-cell beam dynamics simulations with a waveletbased Poisson solver, Phys. Rev. ST Accel. Beams 10 (2007) 034201.

[6] M. Hess, C. Park, A Multi-Slice Approach for electromagnetic Green's function based beam simulations, Proc. 2007 Particle Accelerator Conf., 2007, pp. 3531-3533.

[7] C. Prior, Space charge simulation, AIP Conf. Proc., 693 (2003) 32-37.

[8] E. Nissen, B. Erdelyi, S. Manikonda, Method to extract transfer maps in the presence of space charge in charged particle beams, Conf. Proc. C100523 (2010) 1967-1969.

[9] E. Nissen, B. Erdelyi, A New Paradigm for Modeling, Simulations and analysis of intense beams, Proc. HB2010, Morschach, Switzerland (2010) 534-538.

[10] E. Nissen, B. Erdelyi, S. Manikonda, A self-consistent multi-processor space charge algorithm that is almost embarrassingly parallel, Conf. Proc. C1205201 (2012) 1128-1130.

[11] D. Gonzalez, B. Erdelyi, Charge density estimations with orthogonal polynomials, Conf. Proc. C1205201 (2012) 355-357.

[12] A. J. Dragt, Lie Methods for Nonlinear Dynamics with Applications to Accelerator Physics, unpublished (2013).

[13] M. Berz, Advances in Imaging and Electron Physics, vol. 108, Academic Press, London, 1999.

[14] K. Makino, M. Berz, Cosy infinity version 9, Nucl. Instrum. Methods A 558 (2005) 346-350.

[15] M. G. Duffy, Quadrature over a pyramid or cube of integrands with a singularity at a vertex, SIAM J. Numer. Anal. 19:6 (1982) 1260-1262.

[16] M. G. Bulmer, Principles of Statistics, MIT Press, Cambridge Massachusetts, 1967.

[17] B. G. Lindsay, P. Basak, Moments determine the tail of a distribution (but not much else), The American Statistician 54:4 (2000) 248-251. 
[18] W. Press, S. A. Teukolsky, W. T. Vetterling, B. P. Flannery, Numerical Recipes in C++, Cambridge University Press, New York, 2002.

[19] M. Bassetti, G. Erskine, Closed Expression for the Electrical Field of a Two-Dimensional Gaussian Charge, Tech. Rep. ISR-TH/80-06, CERN (1980).

[20] L. N. Trefethen, D. Bau, Numerical Linear Algebra, Society for Industrial and Applied Mathematics, Philadelphia, 1997.

[21] M. Reiser, Theory and Design of Charged Particle Beams, WILEY-VCH Verlag GmbH \& Co., Weinheim, 2008.

[22] M. Berz, Differential algebraic description of beam dynamics to very high orders, Part. Accel. 24 (1989) 109-124.

[23] A. Griewank, A. Walther, Evaluating Derivatives: Principles and Techniques of Algorithmic Differentiation, Second Edition, Society for Industrial and Applied Mathematics, 2008.

[24] E. Nissen, B. Erdelyi, An Implementation of the fast multipole method for high accuracy particle tracking of intense beams, Conf. Proc. C110328 (2011) 1782-1784. 